
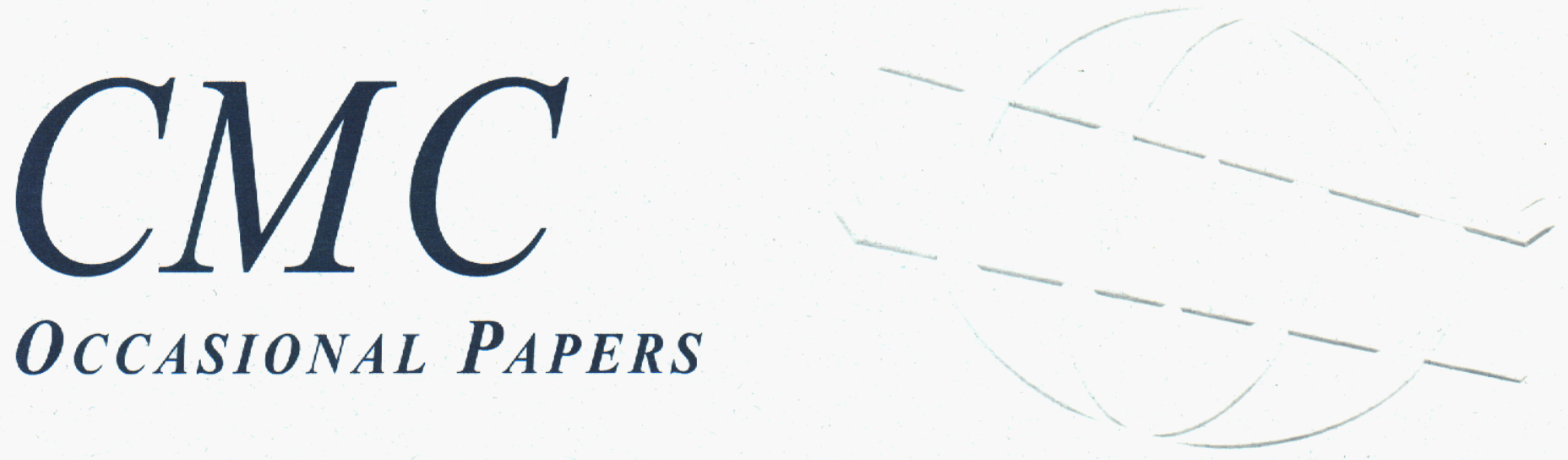

\title{
Concepts for Cooperative Nuclear Monitoring in Bangladesh
}

C. S. Karim, Member, Physical Science Bangladesh Atomic Energy Commission Bangladesh 
Issued by Sandia National Laboratories, operated for the United States Department of Energy by Sandia Corporation.

This report was prepared as an account of work sponsored by an agency of the United States Government. Neither the United States Government nor any agency thereof, nor any of their employees, nor any of their contractors, subcontractors, or their employees, makes any warranty, express or implied, or assumes any legal liability or responsibility for the accuracy, completeness, or usefulness of any information, apparatus, product, or process disclosed, or represents that its use would not infringe privately owned rights. Reference herein to any specific commercial product, process, or service by trade name, trademark, manufacturer, or otherwise, does not necessarily constitute or imply its endorsement, recommendation, or favoring by the United States Government, any agency thereof, or any of their contractors or subcontractors.

The views and opinions expressed herein do not necessarily state or reflect those of the United States Government, any agency thereof, or any of their contractors.

Printed in the United States of America. This report has been reproduced directly from the best available copy.

Available to DOE and DOE contractors from

U.S. Department of Energy

Office of Scientific and Technical Information

P.O. Box 62

Oak Ridge, TN 37831

Telephone:

(865) 576-8401

Facsimile:

(865) 576-5728

E-Mail:

reports@Adonis.osti.gov

Online ordering:

http://www.doe.gov/bridge

Available to the public from

U.S. Department of Commerce

National Technical Information Service

5285 Port Royal Rd.

Springfield, VA 22161

Telephone:

Facsimile:

E-Mail:

Online order:
(800) 553-6847

(703) 605-6900

orders@ntis.fedworld.gov

http://www.ntis.gov/help/ordermethods.asp?loc=7-4\#online 
SAND 2003-0751

March 2003

\title{
Concepts for Cooperative Nuclear Monitoring in Bangladesh
}

\author{
C. S. Karim, Member, Physical Science \\ Bangladesh Atomic Energy Commission \\ Bangladesh
}

Cooperative Monitoring Center Occasional Paper/27

\section{Sandia National Laboratories}

Sandia is a multiprogram laboratory operated by Sandia Corporation, a Lockheed Martin Company, for the United States Department of Energy under contract DE-AC04-94AL85000. 
The Cooperative Monitoring Center (CMC) at Sandia National Laboratories assists political and technical experts from around the world to acquire the technology-based tools they need to assess, design, analyze, and implement nonproliferation, arms control, and other' cooperative security measures. As part of its mission, the CMC sponsors research on cooperative security and the role of technology. Reports of that work are provided through the Occasional Papers series. Research is conducted by Sandia staff, international technical experts, and visiting scholars. (The CMC's Visiting Scholars Program is administered by the Institute for Public Policy at the University of New Mexico.) For additional information on the CMC's programs, visit the CMC home page on the World Wide Web at $<$ http://www.cmc.sandia.gov> or write to:

Cooperative Monitoring Center

Sandia National Laboratories

Mail Stop 1373

Albuquerque, NM 87185-1373

For specific information on this report contact:

John Olsen at the above address.

This report was prepared by Sandia National Laboratories

Albuquerque, NM 87185 and Livermore, CA 94550 


\title{
Concepts for Cooperative Nuclear Monitoring in Bangladesh
}

\begin{abstract}
This paper proposes a nuclear cooperative monitoring project in Bangladesh, implemented through a cooperative arrangement between the Bangladesh Atomic Energy Commission (BAEC) under the Ministry of Science and Information \& Communication Technology, Government of Bangladesh and the Cooperative Monitoring Center at Sandia National Laboratories under the U.S. Department of Energy. The project's intent is to increase nuclear transparency in the South Asia Region in hopes of building confidence in the region. This paper identifies the need for and benefits of the project. The author discusses the project's scope, tasks, and phases as well as related issues concerning implementation, operation, and maintenance.

The proposed monitoring project encompasses three components: (1) sharing of information on the overall nuclear program (i.e., relevant annual reports); (2) nuclear environmental monitoring; and (3) nuclear facility and materials monitoring. In the first component, the cooperative initiative would make currently available information more accessible through a web site. In the second component, available data on environmental monitoring would be reviewed and authenticated before dissemination. Also, the BAEC's nuclear monitoring capabilities would be strengthened through the acquisition of monitoring technology and the application of uniform procedures. New project-developed data would be generated and shared. The third component calls for the installation of sensors and other surveillance equipment in key facilities, such as a research reactor, an isotope production facility, and a radioactive waste management facility. Dissemination of information would enhance nuclear transparency in the region and strengthen existing programs of the International Atomic Energy Agency.

Nuclear transparency in South Asia could help minimize the risks of misinterpretation, increase nuclear stability in times of crises, and ensure the effective management and resolution of crises. Enhanced security of Bangladesh's nuclear facilities and materials could reduce the possibility of the misuse of nuclear materials. The Bangladesh project could be a model for other regions that have nuclear installations, leading to confidence building at regional levels.
\end{abstract}




\section{Acknowledgments}

I gratefully acknowledge the support provided by the Department of Energy, Government of the United States of America and the Ministry of Science and Information \& Communication Technology, Government of the People's Republic of Bangladesh for their support to the project.

The Cooperative Monitoring Center of the Sandia National Laboratories created an atmosphere that was most congenial to the development of the project. CMC itself is a manifestation of transparency through cooperation. I would like to put on record my sincere appreciation of the opportunities offered by the CMC that helped conceptualize and formulate the project. I owe special thanks to Fred Luetters for his encouragement.

I express my sincere gratitude to CMC staff members Kent Biringer, George Baldwin, Amir Mohaghegi, and consultant Gaurav Rajen for their valuable suggestions. Their willingness to share information and experience was instrumental in obtaining a complete picture of what is needed to develop a transparency project. They were the sources of my inspiration, and persons always eager to extend helping hands with a spirit of cooperation.

I also wish to recognize the support provided by other staff members, in particular that by John Olsen, Tim Crawford, Don Glidewell, Richard Lucero, and Suzanne Cordova for their support and for acquainting me with a range of technological options of nuclear transparency.

I thank Stephen Garcia, whose magical touches turned almost incomprehensible diagrams into something presentable.

Eric Whitmore is one of the nicest persons I have ever met. His help was always available and he attended to all my requests (however unreasonable those could be) with a smile.

I have no hesitation in saying that CMC is a great place to work. This has been made possible by the support provided constantly by Patricia Dickens and Stephanie Sustaita. They tackled all problems with an ease that one can't even imagine. With them around, work and life were always pleasant.

C.S. Karim 


\section{Contents}

EXECUTIVE SUMMARY 9

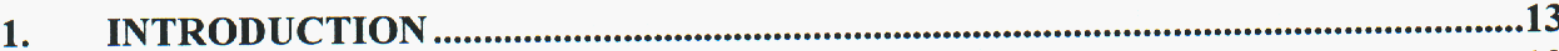

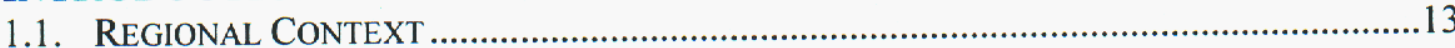

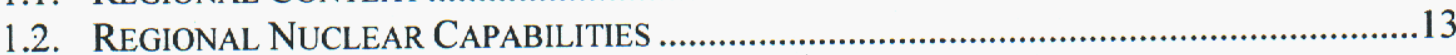

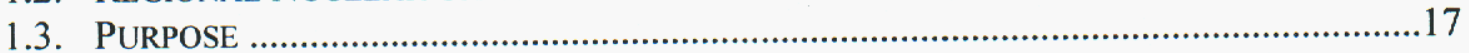

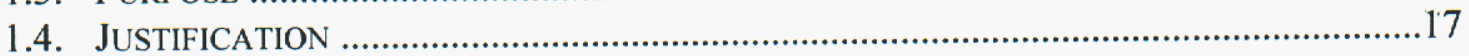

1.5. OBjective AND Possible OUtCOMES OF THE BANGLADESH

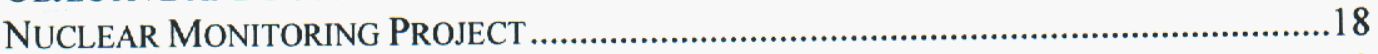

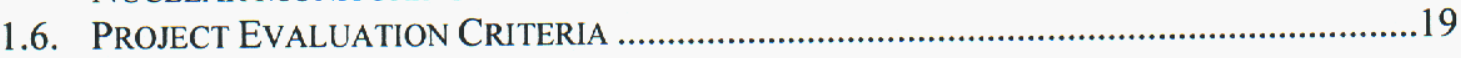

2. DESCRIPTION OF PROJECT ……..................................................................................20

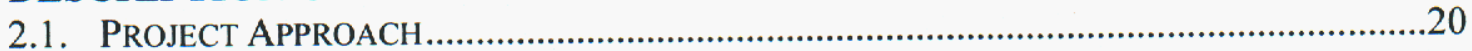

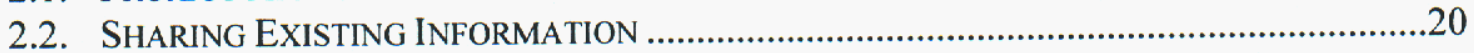

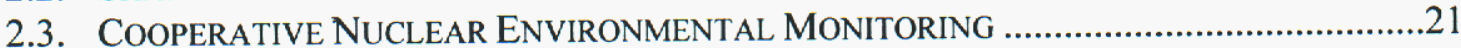

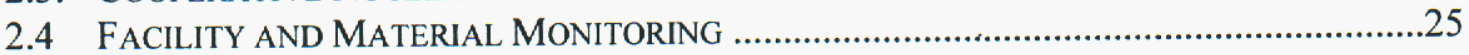

3. SPECIFIC ACTIVITIES.............................................................................................26

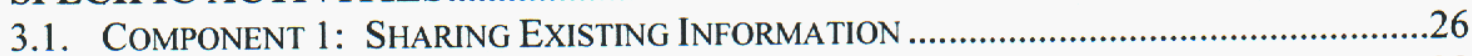

3.2. COMPONENT 2: COOPERATIVE NuClEAR ENVIRONMENTAL MONITORING ..................28

3.3. COMPONENT 3: FACILITY AND MATERIAL MONITORING .............................................

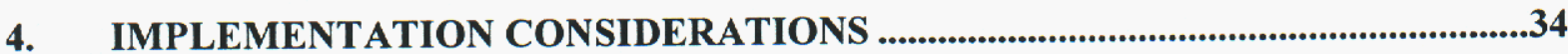

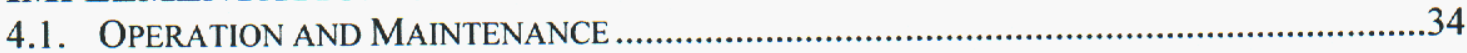

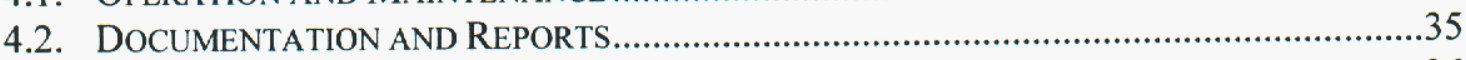

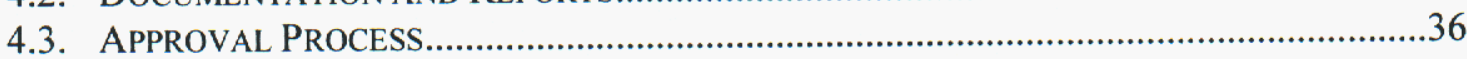

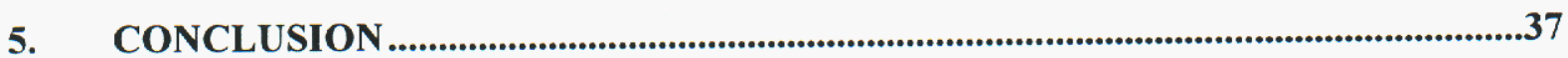

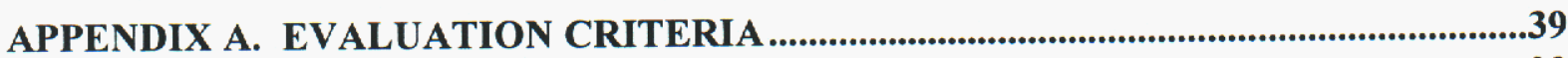

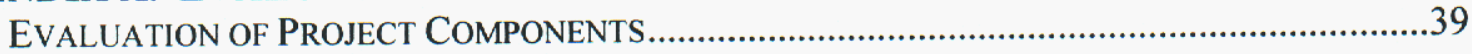

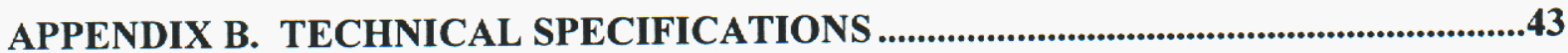

B-1. EQUIPMENT FOR ENVIRONMENTAL MONITORING...................................................43

B-2. FACILITY AND MATERIAL MONITORING.............................................................47

\section{Figures}

Figure 1. Bangladesh and Surrounding Countries of South Asia and Southeast Asia ...............14

Figure 2. BAEC Nuclear Establishments ..........................................................................24

Figure 3: General Plan for CMC/Bangladesh Nuclear Cooperative Monitoring

Implementation. .34 


\section{Acronyms}

$\begin{array}{ll}\text { AERE } & \text { Atomic Energy Research Establishment (Bangladesh) } \\ \text { BAEC } & \text { Bangladesh Atomic Energy Commission } \\ \text { C\&S } & \text { containment and surveillance } \\ \text { CBM } & \text { confidence building measure } \\ \text { CMC } & \text { Cooperative Monitoring Center } \\ \text { CSCAP } & \text { Council on Security Cooperation in the Asia-Pacific } \\ \text { CTBT } & \text { Comprehensive Test Ban Treaty } \\ \text { CVM } & \text { critical Venturi meter } \\ \text { DAS } & \text { data acquisition system } \\ \text { DIRS } & \text { data and image review system } \\ \text { DOE } & \text { Department of Energy (US) } \\ \text { ECNEC } & \text { Executive Committee of the National Economic Council } \\ \text { EML } & \text { Environmental Monitoring Laboratory (US DOE) } \\ \text { EPA } & \text { Environmental Protection Agency } \\ \text { FAST } & \text { Fiber-Optic Attribute Sensor Technology } \\ \text { FTE } & \text { full time equivalent } \\ \text { IAEA } & \text { International Atomic Energy Agency } \\ \text { MDN } & \text { master data node } \\ \text { MOSICT } & \text { Ministry of Science and Information \& Communication Technology } \\ \text { MW } & \text { megawatt } \\ \text { NPT } & \text { Treaty on the Non-proliferation of Nuclear Weapons, also known as the Non- } \\ & \text { Proliferation Treaty } \\ \text { PHWR } & \text { pressurized heavy water reactor } \\ \text { QA } & \text { quality assurance } \\ \text { R\&D } & \text { research and development } \\ \text { RCA } & \text { regional cooperation agreement } \\ \text { RMS } & \text { remote monitoring system } \\ \text { SAARC } & \text { South Asian Association for Regional Cooperation } \\ \text { SNL } & \text { Sandia National Laboratories } \\ \text { SNM } & \text { special nuclear material } \\ \text { VFC } & \text { volumetric flow controller }\end{array}$




\section{Concepts for Cooperative Nuclear Monitoring in Bangladesh}

\section{Executive Summary}

South Asia is a volatile region with a number of contentious bilateral issues. In spite of many historical commonalities, the mutual distrust prevailing among some countries of the region is disquieting. The state of affairs deteriorated further following the nuclear detonations in 1998 by India and Pakistan. There is an urgent need to promote confidence building measures (CBMs) among countries in this region to enhance stability and increase cooperation. The project proposed here could serve as one possible nuclear-related CBM for South Asia.

India and Pakistan have mature programs in nuclear research and development (R\&D), including nuclear power generation and weapons programs. Bangladesh is active in peaceful applications including a plan for nuclear power generation. Sri Lanka also uses nuclear technologies in various fields. Thus, the region is vulnerable to radiation releases from nuclear civilian and weapons-related installations. Being a densely populated region, accidental or deliberate radiation releases in South Asia could present a significant risk to human health. Issues related to the physical security of nuclear materials and the environmental releases of radioactivity are, therefore, of great and common concern to all the countries of South Asia. These common issues could form the basis of regional cooperation projects.

The countries in the region participate in some common nuclear R\&D programs under the Regional Cooperation Agreement of the International Atomic Energy Agency (IAEA). However, sharing data and information related to nuclear transparency is yet to be on the regional agenda. Hence, in the regional context, there is a need to strengthen nuclear transparency and build confidence.

The status of adherence to the international non-proliferation regime in the region varies from country to country. Bangladesh has implemented the full range of the non-proliferation regime including signing and ratifying the Treaty on the Non-proliferation of Nuclear Weapons (NPT) and the Comprehensive Test Ban Treaty (CTBT). Sri Lanka, Nepal, Bhutan, and Maldives are signatories to the NPT and various safeguards agreements with the IAEA. India and Pakistan, on the other hand, have not signed the NPT or the CTBT, and have chosen to have a selective and facility-based approach to nuclear verification and IAEA Safeguards. Many Indian and Pakistani nuclear facilities are not under any international safeguards. In the absence of mutual or international comprehensive monitoring regimes, cooperative monitoring of some of the non-sensitive aspects of each other's nuclear facilities could be a viable approach to enhance nuclear transparency and build confidence among South Asian countries. Because Bangladesh has built advanced nuclear programs that adhere to the international nonproliferation regime, its nuclear facilities present a favorable opportunity for demonstrating cooperative monitoring technologies.

The Bangladesh Atomic Energy Commission (BAEC), under the Ministry of Science and Information \& Communication Technology, is responsible for nuclear-related activities in 
Bangladesh. The BAEC's key installations, located at its complex at Savar, include a 3-MW research reactor, an isotope production facility, and a radioactive waste facility. Radiation technology for medical, industrial, and agricultural applications is used in other locations in the country.

In addition to its strong support for non-proliferation, Bangladesh is also committed to fostering regional stability. After the nuclear detonations in India and Pakistan in 1998, the Bangladesh government played a proactive role in requesting India and Pakistan to show restraint in using their respective nuclear capabilities. Bangladesh also successfully initiated the South Asian Association for Regional Cooperation, which remains one of the few political frameworks in which India and Pakistan consistently engage in dialogue. Such actions distinguish Bangladesh in the region.

In 2000, the Cooperative Monitoring Center (CMC) of Sandia National Laboratories (SNL) initiated collaboration with the BAEC on a proposed cooperative nuclear monitoring project. During a meeting between CMC and BAEC staff in Bangladesh in March 2001, two promising avenues for collaboration were identified: (1) nuclear environmental monitoring, and (2) facility and materials monitoring. These ideas were developed into a specific plan of action during the visit to Sandia by the author in February-April 2002. In a continuation of these efforts, Bangladesh and the $\mathrm{CMC}$ are proposing to initiate a nuclear monitoring project to improve nuclear stability and build trust and confidence in the region.

Initially, this proposed collaboration with the CMC would involve solely Bangladesh entities. Eventually, it is hoped that Bangladesh's efforts to share monitoring data from its nuclear facilities would motivate other countries in the region to participate in the transparency project.

The proposed monitoring project encompasses three components: (1) sharing of information on the overall nuclear program (i.e., relevant annual reports); (2) nuclear environmental monitoring; and (3) nuclear facility and materials monitoring. In the first component, the cooperative initiative would make currently available information more accessible through a web site. In the second component, as an initial step, available data on environmental monitoring would be reviewed and authenticated before dissemination. In a subsequent stage of this component, the infrastructure and capacity of the BAEC in related activities would be strengthened through the acquisition of monitoring technology and the application of uniform procedures, and new project-developed data would be generated and shared, as opposed to the sharing of pre-existing data gathered for separate programs. In the third component, sensors and other surveillance equipment would be installed in key installations, such as the research reactor, the isotope production facility, and the radioactive waste management facility, for capturing data and information on facility and materials status on a continuous basis. Dissemination of information would enhance nuclear transparency in the region and to strengthen existing IAEA verification programs.

The project would strengthen activities in Bangladesh in nuclear verification and environmental monitoring. The project would also establish a precedent for regional practices. In the future, the scope of the cooperative monitoring project can be extended to provide linkages to other similar nuclear transparency projects under way in the Asia-Pacific region, such 
as through the Council on Security Cooperation in the Asia-Pacific (CSCAP) and other conventional environmental monitoring projects under way in South Asia, thereby creating additional mutual benefits.

Nuclear transparency in South Asia could help minimize the risks of misinterpretation, increase nuclear stability in times of crises, and ensure the effective management and resolution of crises. Enhanced security of nuclear facilities and materials would reduce the possibility of the misuse of nuclear materials for destructive purposes in the country or elsewhere.

The Bangladesh project could be a model for other regions that have nuclear installations, leading to confidence building at regional levels. The United States and Bangladesh have signed a nuclear cooperation agreement, which, among other issues, provides for cooperation in the general areas of security of nuclear facilities and materials and safeguards. Bangladesh has the expertise and experience to conduct the envisaged monitoring activities on a sustainable basis. Cooperation between the $\mathrm{CMC}$ and the BAEC at the operation level would have far-reaching benefits for both the parties.

Bangladesh is the only country in the region that is currently agreeable to initiating the proposed project. The project has the potential to assume a regional dimension. At present, there is no other viable proposition to even conceptualize a regional approach to nuclear nonproliferation in South Asia. 
Concepts for Cooperative Nuclear Monitoring in Bangladesh

This page intentionally left blank. 


\section{Concepts for Cooperative Nuclear Monitoring in Bangladesh}

\section{Introduction}

\subsection{Regional Context}

South Asia ${ }^{1}$ (Figure 1) is a volatile region with contentious bilateral issues, occasional political turmoil, and internal and external ethnic clashes. One source of regional tension relates to the effects of nuclear activities and development in South Asia. For example, the construction and operation of new nuclear power stations in India are causes for concern for neighboring Bangladesh. India mines uranium near its border with Bangladesh. In addition, Bangladesh plans to implement its own nuclear power program, which could affect other States in the region.

Of deepest concern is the mutual distrust and antagonism resulting from the conflicts between India and Pakistan. Over a half-century of conflict has created a precarious situation: the possibility of a pre-emptive nuclear first strike followed by retaliation. ${ }^{2}$ It was, however, heartening to note that both countries showed restraint during the Kargil skirmishes, which emerged one year after the May 1998 nuclear tests, when combat was limited to conventional weapons. They again showed restraint in the summer of 2002 when tensions concerning terrorism were high. This mutual restraint provides a glimmer of hope that the nuclear weapon capabilities within South Asia may never be deployed. In 1998, Bangladesh played a proactive role in requesting India and Pakistan to show restraint in using their respective nuclear capabilities. ${ }^{3}$ Nuclear-related confidence building measures (CBMs) could reinforce the nuclear restraint being demonstrated by India and Pakistan. The projects proposed and studied in this paper could serve the role of such nuclear CBMs in South Asia.

\subsection{Regional Nuclear Capabilities}

In South Asia, India, Pakistan, Bangladesh, and Sri Lanka are involved in nuclear-related activities. There is a very wide divergence in their respective national goals, programs, expertise, and infrastructure. The nuclear program in Bangladesh is a legacy of its existence as East Pakistan, and its nuclear activities were initiated simultaneously with those in current Pakistan (formerly West Pakistan). From the time of independence in 1971 and the subsequent formation of the Bangladesh Atomic Energy Commission (BAEC), the intent of Bangladesh's nuclear research and development $(R \& D)$ has focused exclusively on peaceful applications.

1 The term "South Asia" is considered in this report to be the regional grouping made up of the countries (Bangladesh, Bhutan, India, Maldives, Nepal, Pakistan, and Sri Lanka) that comprise the South Asian Association for Regional Cooperation (SAARC).

2 Vijay K. Nair, The Structure of an Indian Deterrent in India's Nuclear Deterrent: Pokhran II and Beyond; Amitabh Matto, ed., Har Anand Publication Ltd., India, 1999.

3 Bangladesh Statement to the IAEA General Conference, 1998. 


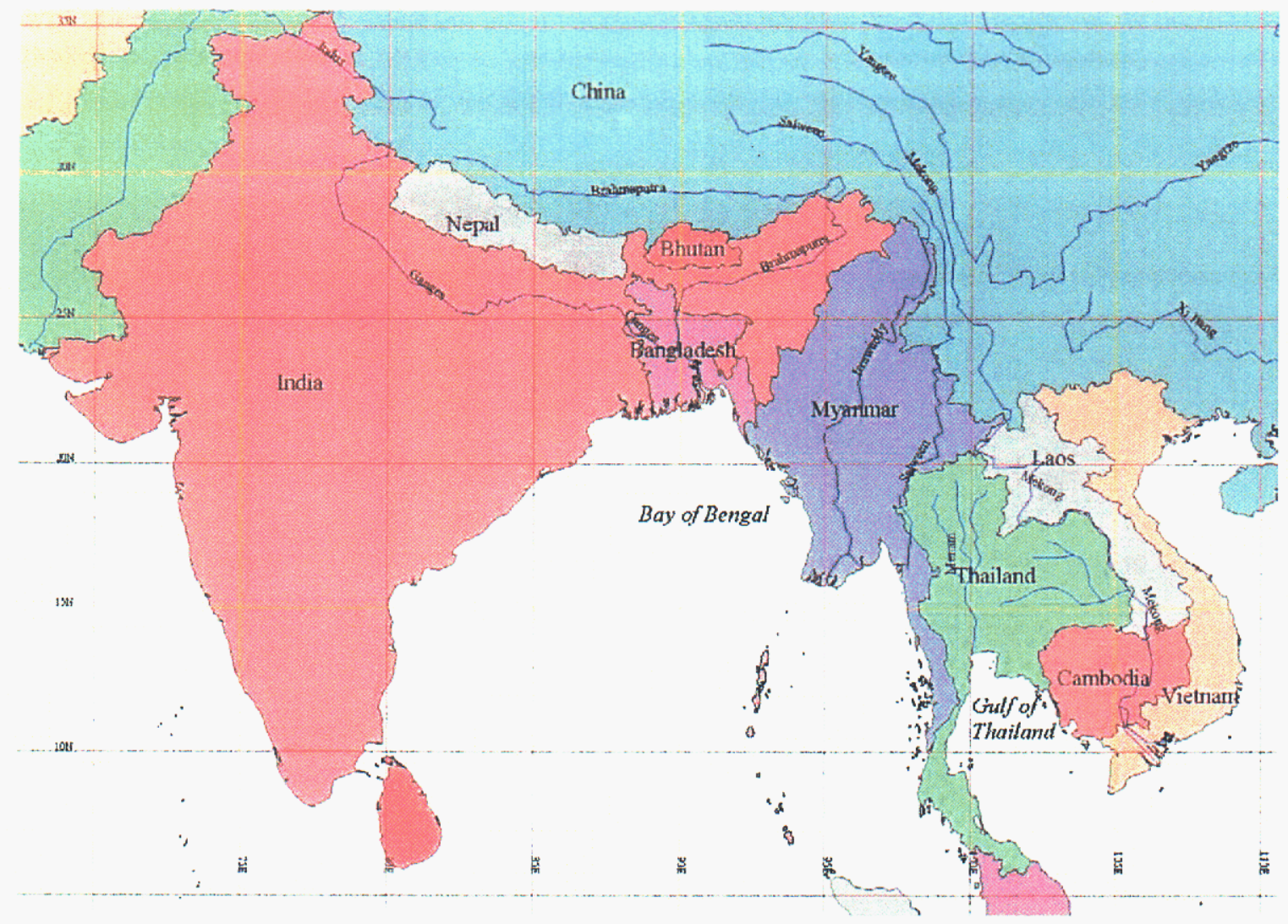

Figure 1. Bangladesh and Surrounding Countries of South Asia and Southeast Asia

Pakistan has implemented a national nuclear weapons program and has developed missile capabilities to deliver warheads. India has mature programs in peaceful and weapons-related applications of nuclear and missile technologies. The use of nuclear technologies in Sri Lanka is limited to their applications in a few selected fields, such as non-destructive testing using nuclear techniques, tracer technology, and radiation technology. ${ }^{4}$ Nepal, Maldives, and Bhutan are not active in the nuclear energy field.

Thus, the region has the potential for the release of radioactivity originating from an accident in a nuclear installation or from a nuclear weapon detonation. In such a complex situation in a region with over 1.3 billion people, the need for reassurance and confidence building assumes overwhelming importance. Nuclear monitoring and transparency can pave the way to such a confidence building process. On the other hand, implementation of a regional monitoring system and transparency has several impediments, including mutual distrust of bilateral and multilateral initiatives. The proposed nuclear monitoring project could be a trendsetter with the expectation that the experience could motivate others in the region to participate in the future.

\footnotetext{
${ }^{4}$ IAEA/RCA website (http://www.rca.iaea.org/SRL/rca2.html.
} 
In the hope that neither India nor Pakistan would take pre-emptive action involving nuclear weapons, this paper has the following premises:

- There is a need to eliminate or reduce tension caused by a lack of confidence.

- Nuclear transparency and cooperation may help build confidence. (See the inset for a discussion on recent changes in attitudes towards nuclear transparency, and the approach adopted in this paper.)

- Confidence building between India and Pakistan may be initiated through a third country in the region, such as Bangladesh.

Except for India and Pakistan, all countries in the region have signed the Treaty on NonProliferation of Nuclear Weapons (NPT) and Bilateral Safeguards Agreements with the International Atomic Energy Agency (IAEA). Pakistan and India, however, have adopted a conditional approach to safeguards, whereby selected facilities (or subparts) are under a bilateral verification program under the auspices of the IAEA. The IAEA's verification program restricts the distribution of results or findings of site inspections to internal reports and the country concerned, which limits the nuclear transparency associated with the IAEA's programs.

Experience shows that the countries in the region are, in some cases, agreeable to sharing non-sensitive data and information. ${ }^{5}$ The technologies involved, the underlying purpose of data sharing, and the sensitivity and relevance of the monitoring project to national interests are some of the factors that can influence the decision on cooperation. The scope of these types of projects evolves over time and depends on bilateral and multilateral relationships. For example, after the commissioning of the Farakka Barrage on the Ganges upstream of Bangladesh territory in the early 1970s, it was almost inconceivable that data on the Ganges could be shared between Bangladesh and India. The barrage on the Ganges upstream of Bangladesh territory is used to divert water from this transboundary river during the lean period, thereby causing problems of availability of water and silt deposits on the riverbed. This became a major contentious issue between the two countries. Data on flow and other parameters were considered sensitive and were thus protected. The change in the approach to data sharing occurred only in the mid-1990s. In light of this possibility of progressively increasing the sharing of sensitive data, a multilateral approach to nuclear transparency could be one option that might be acceptable to all the countries in the region. A nuclear transparency project can start with a single country, such as Bangladesh, and as the system evolves and intentions are made clear, other countries might feel encouraged to participate in the project. The proposed monitoring project could eventually become part of a larger confidence building process.

5 Gaurav Rajen, Kent L. Biringer, and J. David Betsill, The South Asia Cooperative Environmental Monitoring Project: An Effort to Promote Regional Cooperation and Water Quality Data Sharing in South Asia, Cooperative Monitoring Center, Sandia National Laboratories, Albuquerque NM, USA, SAND 2001-0722. 


\section{Transparency: An Evolving Concept}

Nuclear transparency can help build confidence and can be initiated on a unilateral, bilateral, regional, or multilateral basis. For example, India and Pakistan have implemented Bilateral Safeguards Agreements with the IAEA on a selective basis, offering a few facilities, or subparts, for verification. National positions and attitudes towards transparency and verification sometimes change with the evolving national political agenda. In South Africa, the policy changed from a complete blackout of information to a fully developed transparency regime after changes occurred in the domestic political scene.

A State's decision to allow transparency has become more complicated after the terrorist acts of September 11, 2001. While a nuclear weapon has not been used, it is widely feared that the terrorists were or are in the process of acquiring the technology, materials, and human resources to produce a weapon of mass destruction or a "dirty" bomb with high-level nuclear wastes. Policy makers face the quandary that providing sensitive information to the public makes it available to terrorists as well.

With this scenario in mind, the question of nuclear transparency needs some objective rethinking. Information and data must be carefully stratified to avoid creating vulnerabilities. Strategies such as withdrawing sensitive information from the public domain, disguising certain information, artificially delaying the dissemination of data, etc. are receiving serious attention. Hopefully, through compromise, proponents can keep the essential tenet and spirit of transparency unchanged, while meeting the more important requirements of maintaining security and preventing the abuse of knowledge. The approach to transparency needs to answer three basic questions:

1. What data should I share?

2. Who should have access to the data?

3. How do I share the information securely?

In the case of the Bangladesh Nuclear Monitoring Project, the original concept of transparency has evolved since the first meeting in March 2001. Because of the 9/11 terrorist attacks, the project team has revisited the need and scope of cooperative nuclear monitoring. It appears that transparency could even cause more harm than benefits if theft of nuclear material in one country resulted in a destructive incident in another country. The physical security of facilities and materials, therefore, assumes a priority that is higher than the desire for transparency. The possible vulnerabilities that full-scale transparency could cause call for a selective approach to nuclear transparency.

Some of the options for selective data sharing are:

- Share simulated and not-to-scale data-use cartoons instead of actual schematics or maps

- Provide conceptual rather than specific information on strategic areas of a facility

- Provide aggregate data for public consumption

- Delay the release of certain information and provide it in the form of past history (such as annual reports)

- Omit some values

- Withhold data that reveal details of physical protection systems (such as locations of doors, guard stations, and other sensitive features of physical security) 


\subsection{Purpose}

This report proposes a nuclear cooperative monitoring project in Bangladesh, implemented through a cooperative arrangement between the BAEC under the Ministry of Science and Information \& Communication Technology (MOSICT), Government of Bangladesh and the Cooperative Monitoring Center (CMC) at Sandia National Laboratories (SNL) under the U.S. Department of Energy (DOE). The project's intent is to increase nuclear transparency in the South Asia region in hopes of building confidence in the region. This paper identifies the need for and benefits of the project and the project's scope, tasks, and phases and discusses related issues, including implementation, operation, and maintenance.

\subsection{Justification}

Countries in South Asia cooperate under the Regional Cooperation Agreement (RCA) framework of the IAEA. However, the sharing of data and information related to nuclear transparency is yet to be on the regional agenda. Hence, there is a need to strengthen regional nuclear transparency. Cooperative nuclear monitoring is one avenue to enhance and complement the goals of the global nonproliferation regime.

To combat the volatility and distrust among countries in South Asia, a regional monitoring project with transparency used as the enabling mechanism could facilitate confidence building. The envisaged nuclear monitoring project in Bangladesh could establish a precedent for regional practices. In the future, the scope of the cooperative monitoring project could be extended to provide linkages to other similar nuclear transparency projects under way in the Asia-Pacific region, such as through the Council on Security Cooperation in the Asia-Pacific (CSCAP) and other conventional environmental monitoring projects under way in South Asia, thereby creating additional mutual benefits. In its ultimate phase and depending on the success of these initiating activities, a regional cooperative monitoring center could become a reality.

Since the incidents of September 11, 2001, international concern for the security of nuclear facilities and materials has assumed more importance, especially as materials stolen from even a research reactor or a waste storage facility could be targeted to a distant country. The Bangladesh project could be a trendsetter for implementing similar projects in other regions with nuclear installations, leading to confidence building at regional levels.

With over four decades of active development of nuclear capability, Bangladesh is an example of a state that adheres to all accepted norms of the global nuclear nonproliferation regime, including full-scope IAEA safeguards under the NPT, the Protocol Additional to IAEA Safeguards, and the Comprehensive Test Ban Treaty (CTBT). Other states in the region do not yet participate fully in the nonproliferation regime. For example, India and Pakistan have not signed the NPT or the CTBT; however, they do accept IAEA safeguards on particular facilities or systems.

Ongoing and future nuclear activities in the region can produce effects that extend beyond State boundaries. Examples include releases of airborne radiation from nuclear installations, fallout from nuclear weapons testing, accidents, deliberate use, pollution from 
uranium mining operations, accumulation of spent reactor fuels and radioactive wastes, radioactivity from secondary coolant discharges into the sea or transboundary rivers, and theft of materials or sabotage of facilities. Such interdependence increases the importance of the regional approach to transparency. As a densely populated region, South Asia is especially concerned about vulnerabilities in physical security and environmental protection.

The BAEC is responsible for nuclear-related activities in Bangladesh. Its key installations, located at its complex at Savar, include a 3-MW TRIGA research reactor, an isotope production facility, and a radioactive waste facility. Radiation technology for medical, industrial, and agricultural use are also applied in other locations in the country. Thus, a number of opportunities exist for nuclear monitoring in Bangladesh.

The project would strengthen activities in Bangladesh in nuclear verification and environmental monitoring. The spin-off benefits at the national level would include capacity building in monitoring, acquiring reliable background information on the radiation loading of the environment, supplementing safeguards obligations, and tracking materials.

The United States and Bangladesh have signed a nuclear cooperation agreement, which, among other issues, provides for cooperation in the general areas of security of nuclear facilities and materials and safeguards. Bangladesh has the expertise and experience to conduct the proposed monitoring activities on a sustainable basis. Cooperation between the CMC and the $\mathrm{BAEC}$ at the operation level would have far-reaching benefits for both the parties.

\subsection{Objective and Possible Outcomes of the Bangladesh Nuclear Monitoring Project}

The cardinal objective of the proposed cooperative nuclear monitoring project is to demonstrate a practical example of nuclear transparency in South Asia.

The possible outcomes of transparency through cooperative monitoring are as follows:

- Building/enhancing confidence and creating awareness at the national level

- Building/enhancing confidence at the regional level (South Asia), thereby reducing regional tensions

- Cooperating with other countries on issues of nuclear transparency on an extended regional basis (such as security cooperation in the Asia-Pacific region)

- Obtaining data and information on nuclear accidents and resulting radiation release to the environment in the region through a regional monitoring network and using such information in evolving emergency action plans to mitigate the effects

- Addressing the needs for further enhancing national credibility with regard to the international non-proliferation regime 


\subsection{Project Evaluation Criteria}

Criteria were developed for ranking candidate projects with respect to their relevance and importance to the overall objectives and goals of the project. Appendix A contains the full evaluation criteria. In summary, the criteria were divided into the required and the desired acceptance criteria, as follows:

\subsubsection{Required Criteria}

The required criteria include the following:

- Conforming to non-proliferation agreements or protocols

- Capabilities and features that demonstrate regional nuclear transparency

- Of interest to, acceptable to, and involving both the BAEC and the CMC

- Employing established and compatible technologies and using readily available consumables, spare parts, etc.

- Compatible with environmental conditions in Bangladesh (e.g., temperature, humidity)

- Reasonably short implementation time

- Implementable, operable, and maintainable locally

- Sustainable operation and long operating life with optimal operating cost

- Minimal potential negative consequences or effects

\subsubsection{Desired Criteria}

The desired criteria include the following:

- Potential for involving other regional and international agencies in funding and the exchange of information and dața on nuclear transparency

- Tangible spin-off benefits to the collaborating parties

- Capable of being extended in the future to a regional or international network

- The system and equipment are cost-optimal

- Expandable to additional nuclear monitoring applications

- Ability to include the system as a part of a comprehensive project on nuclear transparency and non-proliferation

- Technology transfer and adoption by the recipient

- Sandia National Laboratories can supply required equipment and systems 


\section{Description of Project}

\subsection{Project Approach}

The important event leading to the conceptualization of a cooperative nuclear monitoring project for Bangladesh was a visit to that country by a three-member team from the CMC in March 2001. The issues of mutual interest were identified initially as follows:

(a) Environmental Monitoring,

(b) Facilities and Materials, and

(c) Regional and Cross-Boundary issues.

Preliminary identification of concepts and technologies related to cooperative nuclear monitoring emerged from discussions of these issues, resulting in the development of a Cooperative Nuclear Monitoring Project for Bangladesh. It is envisaged that the proposed elements of the project would be implemented in phases, beginning with the one that would be comparatively easy to implement, entail limited resources and money, but would have immediate results and visibility. The duration of the initial project cycle is estimated to be three years, followed by an extended period of its operation and maintenance and information sharing.

The nuclear monitoring activities are planned to encompass three broad areas:

1. Sharing Existing Information-Survey existing general information sources at the BAEC and the CMC and select items that could benefit both organizations as well as the public and provide the information on a global-access web site.

2. Cooperative Nuclear Environmental Monitoring-Use the current BAEC infrastructure to provide nuclear monitoring data from air, water, soil, and vegetation samples. Share information as appropriate and choose a subset of information for release to the public. For example, airborne radioactivity levels could be shared with the public via the web site to build confidence in the safety of nearby reactors. The BAEC has experience in these types of monitoring activities.

3. Facility and Materials Monitoring-Approved and carefully chosen information about reactor operations and physical security information would be released to the BAEC or CMC. A limited subset (see the inset on Transparency: An Evolving Concept) of information might be made available on the public web site.

The activities provide a phased approach to nuclear monitoring and transparency, with the long-term goal of involving other countries in the region.

\subsection{Sharing Existing Information}

Existing data and information such as annual reports, descriptions of key R\&D programs, operating experiences, etc., may be shared. This approach may help in launching the cooperative monitoring activities in a relatively short time frame. 


\subsection{Cooperative Nuclear Environmental Monitoring}

The BAEC has some experience and resources (i.e., facilities and trained personnel) in the field of nuclear environmental monitoring. The ongoing BAEC activities may be expanded and authenticated data and/or information may be made available through a network as a part of transparency. The main advantage of this approach as an initial activity of cooperative monitoring is the BAEC's pre-existing expertise and the ready availability of some data.

This portion of the nuclear environmental project may be split into the following phases.

- Phase 1: Sharing of existing data and information on nuclear environmental monitoring

Data and information selected for sharing must meet some general conditions of quality, reliability, and consistency to ensure that the project goals of transparency and confidence building are met.

- Phase 2: Strengthening activities on nuclear environmental monitoring and the collection of new project-developed data

BAEC projects on nuclear environmental monitoring should be strengthened to make the BAEC's expertise and infrastructure compatible with state-of-the-art systems. This may proceed with the appraisal of the existing project and by examining various features and capabilities of the BAEC. This includes the identification of:

- Strengths and weaknesses of the present activities and facilities

- Current procedures and areas needing improvement

- Additional measures including technological inputs needed to upgrade the existing system

- Technology needed for networking (i.e., BAEC/CMC communications)

\subsubsection{Protocol and Procedures}

A comprehensive protocol should be developed to address the following:

- Collecting samples

- Defining the procedures and methodology for analysis including needed equipment and facilities

- Selecting equipment for sampling and analysis

- Creating a quality assurance (QA) program that includes cross verification (for example, participating in the QA Program of the DOE Environmental Monitoring Laboratory). 


\subsubsection{Scope of Environmental Monitoring}

At present, environmental samples (other than air) are collected from 64 administrative districts of the country. The following laboratories could be used for additional projectdeveloped sample collection:

- Eleven nuclear medicine centers of the BAEC

- Beach Sand Exploration Center, Cox's Bazaar

- Site Office for Rooppur Nuclear Power Project

Sample collection would require a uniform protocol to be developed for the purpose. The infrastructure and capacity of the BAEC would require enhancements to enable the systematic survey and analysis of various types of samples.

Sample analysis may be distributed between the Atomic Energy Center, Dhaka and the Institute of Nuclear Science and Technology. The Radiation Testing and Monitoring Laboratory at Chittagong may concentrate on sampling and analysis of marine and coastal water.

The importance of sampling marine and coastal water and benchmarking its background radioactivity is evident from the following:

- Some Indian nuclear power plants are located along the coast of the Bay of Bengal and use sea water as their respective ultimate heat sinks; others are located along transboundary rivers that flow into the Bay of Bengal. ${ }^{6}$

- The beach sand along the coastline has comparatively higher content of certain types of heavy and radioactive minerals, such as illmenite, magnetite, rutile, monazite, kyanite, zircon, etc. $^{7}$

There is a need for reliable background radiation measurements, mapping, and analysis for samples of air, water (including surface, ground, coastal, and rain water), soil, sediment, vegetation, food chain elements (such as cereals, milk, meat, and fish), and various bioassays.

The desired analysis parameters would include gamma spectrum, gross alpha, gross beta, tritium, and uranium and thorium isotopic analysis.

6 The Madras Atomic Power Station, for example, is located along India's east coast near the city of Chennai with two 170-MWe pressurized heavy water reactors (PHWRs) that are located along the southern edges of the Bay of Bengal. The Narora Atomic Power Station has two 220-MWe PHWRs and is located in the state of Uttar Pradesh. Coolant water discharges from this site could affect the Ganges River, which flows into Bangladesh and into the Bay of Bengal. For details of these power plants, see the web site of India's Department of Atomic Energy: http://www.dae.gov.in/.

7 The Beach Sand Exploration Center at Cox's Bazaar has studied the characteristics of Bangladesh's beach sands and is working to extract heavy minerals from these sands. See http://www.rca.iaea.org/BGD/BAEC\%20 Latest $\% 20 \mathrm{WelCome} \% 20 \mathrm{Page} \% 20$.htm. 
The infrastructure and capacity of the BAEC require enhancements to enable the staff to conduct systematic surveys and analysis of various types of samples.

For water, soil, and biological analyses, samples would be collected from designated places in the country through various BAEC establishments. The analysis would be conducted in three laboratories situated at Atomic Energy Research Establishment (AERE), Savar; the Atomic Energy Center, Dhaka; and the Radiation Testing and Monitoring Laboratory, Chittagong. BAEC establishments are located at all these places, which could help optimize the cost of operation and maintenance.

The main objective for establishing the air sampling data stations is to obtain information on background airborne radiation in Bangladesh. Such monitoring would help assess the changes resulting from accidental release of radiation from a facility within the territorial limits of the country or beyond it. At least six such stations could be established in the following areas of the country (Figure 2):

- Savar: An air-monitoring unit near the research reactor would help assess the airborne radioactivity in and around the reactor (e.g., for an area within a $10 \mathrm{~km}$ radius). This would provide the benchmark measurement and monitor potential airborne radioactive releases from the facility.

- Khulna (Southwest): Proximity of this site to the Sunderbans mangrove forest (entered on the World Heritage List of the United Nations Educational, Scientific, and Cultural Organization) would make the data useful, especially because a new nuclear power plant may be built by India near this region.

- Rooppur (Midwest): The site selected for the planned nuclear power plant in the district of Pabna. Benchmark information on background radiation would help assess the possible radiological implications of building the proposed power plant at this site.

- Sylhet (Northeast), Rangpur (Northwest), and Chittagong (Southeast): Capturing data on airborne radiation resulting from nuclear accidents or nuclear weapon projects originating beyond the nation's borders.

\subsubsection{Frequency of Environmental Sampling}

Considering the seasonal variations and the cost of off-line sampling and monitoring, it would be useful to collect environmental samples of soil, water, and vegetation twice a year, i.e., during May to September (monsoon) and December to April (dry months). These two sets of data from each data station would incorporate the major seasonal variations. In the beginning, however, it would be useful to collect four sets of data for a year, which could be used for future reference and benchmarking.

Air samples would be collected and analyzed continuously (with the exception of maintenance time, i.e., allowing time to change filters). 
BAIGLADESH ATOMAC EIIERG COMUMSSSOH ESTABLISHMEHTS

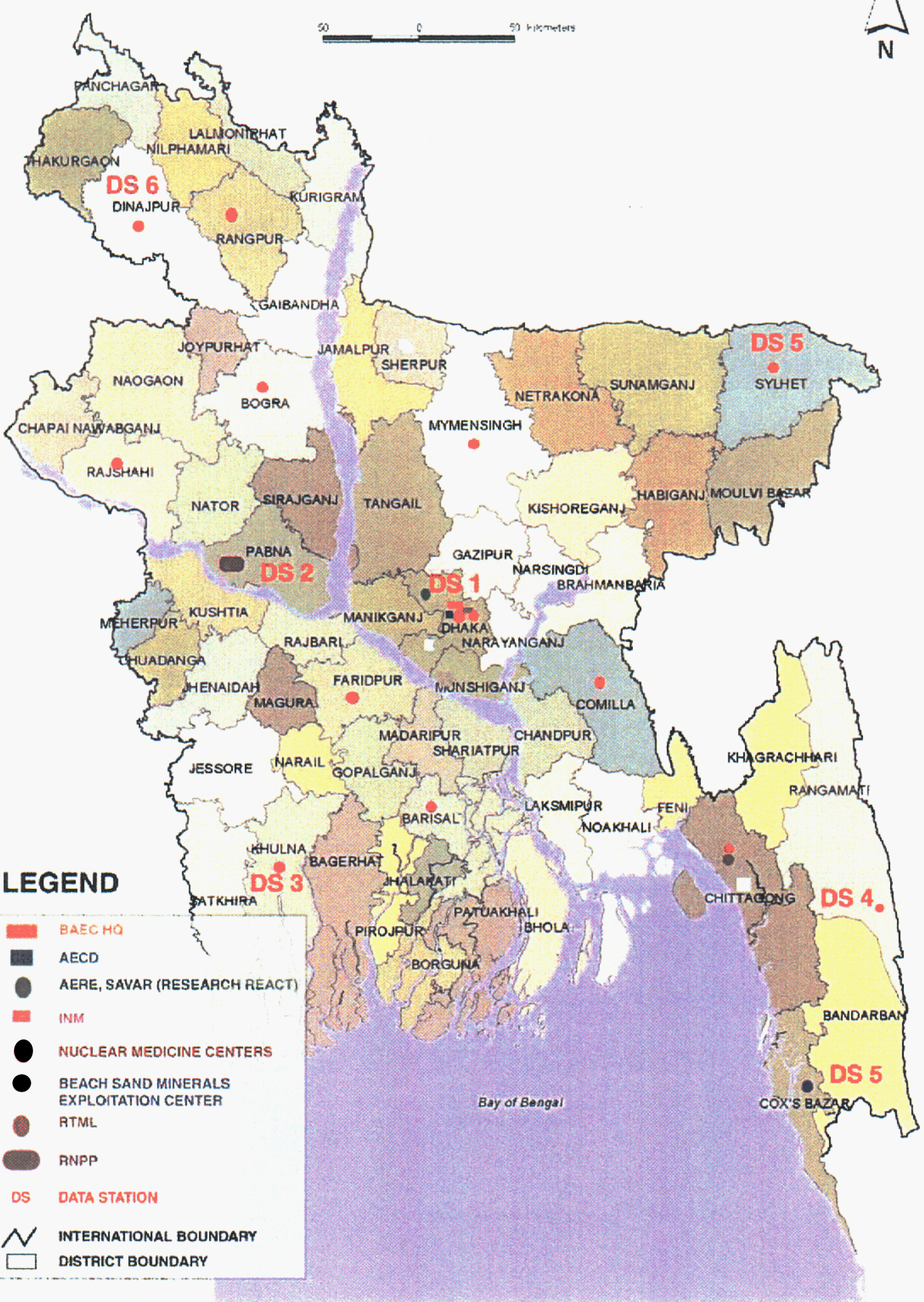

Figure 2. BAEC Nuclear Establishments 


\subsection{Facility and Material Monitoring}

Key surveillance areas include the reactor, fresh and spent fuel storage pits, the ventilation system/stack, radioactive waste management and intermediate storage facility, and isotope production, etc. ${ }^{8}$ The identified types of monitoring are:

- Access monitoring,

- Access control and physical security,

- Exchange of information on operation, abnormal situations/events, and utilization of research reactor, and

- In-house material accounting/monitoring on a continuous basis to supplement periodic inspections by the IAEA.

\section{Atomic Energy Research Establishment, Savar}

The three facilities and associated areas at the Atomic Energy Research Establishment (AERE) in Savar considered most suitable for enhanced containment and surveillance monitoring projects are the:

(a) Research Reactor Facility, including fresh and spent fuel storage, the control room, the reactor core, and the top of the reactor pool.

(b) Radioactive Waste Management Laboratory, including temporary storage of radioactive wastes.

(c) Isotope Production Laboratory, including inventory of imported Molybdenum-99, material balancing, and glove boxes.

Areas of interest include material identification, material balancing, physical security, safety issues, and radiation control. A variety of technological options for monitoring, surveillance, tamper indication, and imaging (continuous, intermittent, or triggered) are available for conducting a comprehensive containment and surveillance project.

8 The facilities identified here need monitoring from both a management perspective and to enhance their physical security and safety. It does not automatically mean that all the data and information would be relevant to the project proposed in this document. 


\section{Specific Activities}

The project approach is to use the existing infrastructure at facilities in the BAEC and the resources available at the $\mathrm{CMC}$ to provide relevant monitoring information to the partners and a limited subset of that information to the public via the Internet to enhance nuclear transparency and build confidence.

The project has three main components described previously in Section 2.1. In this chapter, proposed activities related to each of these components are described in greater detail.

\subsection{Component 1: Sharing Existing Information}

\subsubsection{Objective}

The objective of the first component is for the BAEC and the CMC to exchange sets of existing information on a regular basis.

\subsubsection{Description}

The general types of information to be shared could include the following:

- Lists of BAEC establishments and other organizational matters

- A description of the overall nuclear R\&D program, including Bangladesh's commitment to the nonproliferation and verification regime

- Extracts from past annual reports

- Sample annual report on the research reactor ${ }^{9}$

- A status paper on the national plan on the introduction of nuclear power

- Past publications on environmental nuclear monitoring (references and abstracts)

- Publications on issues relevant to the use of nuclear technology

- Publications available from the $\mathrm{CMC}$

- Description of the experience and expertise of the CMC in remote monitoring projects

- Monitoring systems and equipment available through the $\mathrm{CMC}$

- Results obtained from selected cooperating partners of the CMC (with the partners' approval)

9 The research reactor was not in full operation for some years. Thus, a representative annual report would be more relevant. As the program progresses, updated annual reports may also be included. 
- Support that may be available from the $\mathrm{CMC}$ in establishing remote nuclear monitoring systems, etc.

The information would be disseminated through a public web site. Initially the web site would include the items listed above, as well as a short description on the objectives and goals of the nuclear monitoring project. Information could be added progressively, such as the status of the treaties, agreements, protocols, etc., related to safeguards and verification related to the countries in the region.

On the web site, links to the following would be provided:

- Existing regional institutions and web sites relevant to nuclear activities, institutions, nuclear programs, facilities, etc.

- $\mathrm{CMC}$ web site

- Future web site of the BAEC

- Web sites of other nuclear-related agencies who may be encouraged to provide links

- Other relevant web sites on Safeguards (i.e., the IAEA)

- Regional cooperative conventional monitoring projects (such as water) implemented through cooperation with the $\mathrm{CMC}$

The exchange of existing public or semi-public information has several advantages: the information is immediately available, has a low level of sensitivity, and has low implementation costs.

\subsubsection{Activities}

Activities under this component would be the exchange of currently available information on nuclear-related activities, which would enable quick implementation. The only time-critical item is the development, testing, and maintenance of the web site.

\begin{tabular}{|c|l|l|}
\hline Activity & \multicolumn{1}{|c|}{ Description } & \multicolumn{1}{|c|}{ Responsibility } \\
\hline 1 & Select materials, reports, information, etc., for exchange & CMC and BAEC \\
\hline 2 & $\begin{array}{l}\text { Design the web site for the Nuclear Monitoring Project of } \\
\text { Bangladesh }\end{array}$ & CMC and BAEC \\
\hline 3 & Implement and launch web site & CMC and BAEC \\
\hline \multicolumn{2}{|l}{ Time frame: Three months from initiation of the cooperative monitoring project. } \\
\hline
\end{tabular}




\subsubsection{Output of Component 1}

1. Availability of data and information on a web site to enhance nuclear transparency.

2. A path created for facilitating regional/international cooperation. The web site could motivate voluntary participation by other countries in the region. With additional participants, the web site could eventually become a tool for enhancing nuclear transparency on a regional or international basis.

\subsection{Component 2: Cooperative Nuclear Environmental Monitoring}

\subsubsection{Objective - Phase 1: Sharing existing information on nuclear environmental monitoring}

The objective of Phase 1 of this component is to share existing data and information on nuclear environmental monitoring.

\subsubsection{Description}

BAEC has been active in nuclear environmental monitoring for many years. Initial efforts concentrated on obtaining background radiation levels. The scope was enhanced following the installation of the research reactor and the accident at Chernobyl. Analysis is conducted on a variety of air, water, soil, and vegetation samples. Some of the investigations form parts of a regional project as well as coordinated research programs of the IAEA. The studies are conducted in three different laboratories of the BAEC. The objectives of the studies are different and therefore use different procedures and QA procedures. The equipment, procedures, and QA program also vary from one laboratory to the other as well as from one project to the other. Some of the data conforms to a given international standard, which can serve as the basis for exchange at the initial stage of the cooperative monitoring project. Before these are made available for multilateral sharing, it is essential that their quality and reliability be assessed.

The partners would need to address the following issues:

(a) Status of the existing environmental monitoring activities of the BAEC

(b) Equipment and facility in use and their adequacy

(c) Procedures and methodology

(d) Quality and reliability of data

(e) Relevance of the data to the objectives of the cooperative monitoring project

The exchange of existing nuclear environmental monitoring data has several advantages: the information is immediately available, has a low level of sensitivity, and is low cost. 


\subsubsection{Activities}

Data that meet requirements for quality and reliability would be selected for sharing. A standard format would be developed jointly by the CMC and the BAEC. In parallel, the CMC may share available data from its projects that relate to nuclear environmental monitoring.

\begin{tabular}{|c|l|l|}
\hline Activity & \multicolumn{1}{|c|}{ Description } & \multicolumn{1}{|c|}{ Responsibility } \\
\hline 1 & Develop a format for evaluation of data & CMC and BAEC \\
\hline 2 & $\begin{array}{l}\text { Provide identified data for dissemination through the web } \\
\text { site }\end{array}$ & BAEC \\
\hline 3 & Evaluate existing data & CMC and BAEC \\
\hline \multicolumn{2}{|l|}{ Time frame: Six months from initiation of the cooperative monitoring project. } \\
\hline
\end{tabular}

\subsubsection{Output of Component 2, Phase 1}

1. BAEC environmental data validated with respect to their relevance to nuclear transparency.

2. Reliable environmental monitoring data available for dissemination through a web site.

3. A test bed for the second phase of sharing new project-developed data on environmental monitoring.

\subsubsection{Objective - Phase 2: Strengthening nuclear environmental monitoring and sample collection and sharing of project-developed data}

The objective of Phase 2, Component 2 is to enhance the capacity of the BAEC in nuclear environmental monitoring and to make new project-developed data and information available for use at the national level and for dissemination though the web site.

\subsubsection{Description}

Some new equipment and facilities would be needed to strengthen the capabilities and capacity of the BAEC in the field of nuclear environmental monitoring and to conduct the studies on a sustainable basis. It is proposed that the CMC would provide the needed equipment and the BAEC would provide administrative resources for installation, infrastructure, and facility upgrades, including upgrading the liquid nitrogen production unit. The table below describes the enhanced facilities and the new equipment needed. Appendix B provides details of the needed equipment.

\begin{tabular}{|c|l|}
\hline Quantity & \multicolumn{1}{|c|}{ CMC-Provided Item (Description) } \\
\hline 2 & $\begin{array}{l}\text { Gamma Spectroscopy (40\% HPGe, p-type, amplifier, ADC, interface board, PC, and } \\
\text { software) HPGe Detectors }\end{array}$ \\
\hline 1 & $\begin{array}{l}\text { Integrated Alpha Spec System (24 chambers with surface barrier detectors, processing } \\
\text { electronics, vacuum system, interface board, PC, and software) }\end{array}$ \\
\hline 1 & Liquid Scintillation Counter ( $\mathrm{H}-3$, alpha/beta separation capability, sample changer) \\
\hline 6 & PM-10 Large volume air sampling stations \\
\hline
\end{tabular}




\subsubsection{Activities}

The activities for implementing this component would involve developing a sampling protocol that would be agreed to by all parties, and then a series of sampling actions as described in the protocol.

The protocol should address the following:

- Defining the data quality objectives (i.e., chain-of-custody requirements, types of analysis, detection limits, turn-around times, analytical methods, and reporting formats and procedures)

- Sample collection and field preparation, if any, before analysis

- Sample receipt and storage by the laboratory

- Sample preparation and radiochemistry

- Sample counting, analysis, and reporting

- Data review and validation

- QA, including cross verification (for example, participating in the QA program at the U.S. DOE's Environmental Monitoring Laboratory (EML), quality control (e.g., instrument checks), independent analysis of a subset of the samples through SNL, procedures, and training, etc.) Links would be established with the EML on collaboration in quality control (blind samples or cross verification of results).

The following actions are to be initiated for implementing the above concepts:

\begin{tabular}{|c|l|l|}
\hline Activity & \multicolumn{1}{|c|}{ Description } & \multicolumn{1}{|c|}{ Responsibility } \\
\hline 1 & $\begin{array}{l}\text { Review BAEC's existing environmental monitoring } \\
\text { program and identify areas of for improvement }\end{array}$ & CMC and BAEC \\
\hline 2 & Identify monitoring required & CMC and BAEC \\
\hline 3 & $\begin{array}{l}\text { Identify equipment/facilities (including the preparation of } \\
\text { technical specification) required for establishing a } \\
\text { complete, state-of-the-art nuclear environmental } \\
\text { monitoring system }\end{array}$ & CMC and BAEC \\
\hline 4 & Determine the scope and identify sampling locations & CMC and BAEC \\
\hline 5 & Develop a protocol (following the practices of DOE's EML) & CMC and BAEC \\
\hline 6 & $\begin{array}{l}\text { Establish linkages with DOE's EML. Collaborate on } \\
\text { Quality Control (i.e., blind samples or cross verification of } \\
\text { results) }\end{array}$ & CMC and BAEC \\
\hline 7 & Evaluate technologies & CMC and BAEC \\
\hline 8 & Procure and install initial monitoring system & CMC and BAEC \\
\hline 9 & $\begin{array}{l}\text { Identify options and determine preferred methods for data } \\
\text { capturing, authentication, and transmittal }\end{array}$ & CMC and BAEC \\
\hline 10 & Determine the scope for data and information exchange & CMC and BAEC \\
\hline 11 & Provide periodic project reports & BAEC \\
\hline Time frame: One year from the initiation of the cooperative monitoring project. \\
\hline
\end{tabular}




\subsubsection{Output of Phase 2, Component 2}

1. Enhanced technical capabilities of Bangladesh in nuclear environmental monitoring.

2. Reliable, quality data on the background radiation in water, soil, coastal areas, and air, which could lead to the development of a state-of-the-art national mapping of background radiation levels.

3. Capability to capture radiation levels and migration paths resulting from routine or accidental releases from nuclear facilities within the country or beyond its borders.

4. Data available for the preparation of emergency response plans.

5. Data available for future studies of the effects of uranium mining in the country or beyond its borders.

6. Sharing data, leading to enhanced nuclear transparency.

\subsection{Component 3: Facility and Material Monitoring}

\subsubsection{Objective}

The objective of the third component is to enhance the BAEC's capabilities in safeguards and physical protection monitoring and to make available data and information for use at the national level and for a subset of the data and information to be disseminated though the public web site.

\subsubsection{Description}

Key nuclear installations and special nuclear material (SNM) located at the AERE in Savar would require additional technology for monitoring of materials and of access to sensitive areas. These technologies would enhance the physical security of nuclear facilities and materials. The primary data received from the selected locations would be processed and transmitted to off-site locations. A subset of carefully selected and screened data could be shared through the web site. The data and information to be collected is divided into the following modes based on their respective goals.

- Mode 1: Nuclear Transparency: Data and information relevant to the proposed nuclear monitoring project that may be shared to enhance nuclear transparency and confidence building at the regional as well as multilateral levels.

- Mode 2: Multilateral Nuclear Safeguards: Data and information that may supplement international verification activities.

- Mode 3: Physical security, safety, radiation protection, and internal management: Data and information that is required for improving physical security (protection), nuclear safety 
and radiation control (confidence building at the national level); and data and information that are required by the BAEC from management perspectives.

Additional equipment and enhanced facilities would be needed for Component 3. (See Appendix B for detailed information.) The on-site equipment needed would include the following:

- Item monitoring and access control monitoring systems including seals and tags, SNM attribute determination, intrusion detectors, and video assessment.

- Computer systems for data acquisition, processing and storage, data and image review, and data transmission

The candidate facility for monitoring would be the 3-MW TRIGA MARK II Research Reactor located at the AERE, Savar. The project would focus on portal and area monitoring (entrances and exits) and monitoring inventories of spent nuclear fuel, SNM, and other radiological materials.

\subsubsection{Activities}

The activities for this component would involve the design of the containment and surveillance system and the implementation of the design. Detailed designs would be developed through consultations between the BAEC and the CMC staff. Key design aspects that must be considered are listed below:

- Local conditions (e.g., power grid conditions; rainfall and high humidity, outdoor temperature (i.e., up to $45^{\circ} \mathrm{C}$ )

- Maintainability of equipment and prospects of sustainable supply of spares and consumables

- Reliable backup power supply

- Microsoft Windows-based software preferred

- Relatively weak infrastructure (unstable and unreliable grid power; weak telecommunication links and background noise) 
The following activities would implement this component of the project:

\begin{tabular}{|c|l|l|}
\hline Activity & \multicolumn{1}{|c|}{ Description } & \multicolumn{1}{c|}{ Responsibility } \\
\hline 1 & Evaluate technologies & CMC and BAEC \\
\hline 2 & Select on-site equipment; develop maintenance manuals & CMC and BAEC \\
\hline 3 & $\begin{array}{l}\text { Define QA program encompassing the installation, } \\
\text { operation, and maintenance phases, etc. }\end{array}$ & CMC and BAEC \\
\hline 4 & Procure and install equipment & CMC and BAEC \\
\hline 5 & $\begin{array}{l}\text { Identify options and determine modes for data capture, } \\
\text { authentication, and transmittal }\end{array}$ & CMC and BAEC \\
\hline 6 & Determine the scope for data/information exchanges & CMC and BAEC \\
\hline 7 & Write periodic reports & BAEC \\
\hline 8 & Write project completion report & CMC and BAEC \\
\hline \multicolumn{2}{|c|}{ Time frame: Three years from the initiation of the cooperative monitoring project. } \\
\hline
\end{tabular}

\subsubsection{Output of Component 3}

1. Enhanced technical capabilities of Bangladesh in the field of containment and surveillance of nuclear facilities and SNMs.

2. Nuclear material balancing reports available on a continuous basis, thereby supplementing annual IAEA Safeguards inspections.

3. Enhanced physical security of facilities and materials.

4. Creation of a database of information of importance to national security.

5. Sharing of data and information, thereby enhancing transparency. 


\section{Implementation Considerations}

Figure 3 presents a general plan for implementation of the project.

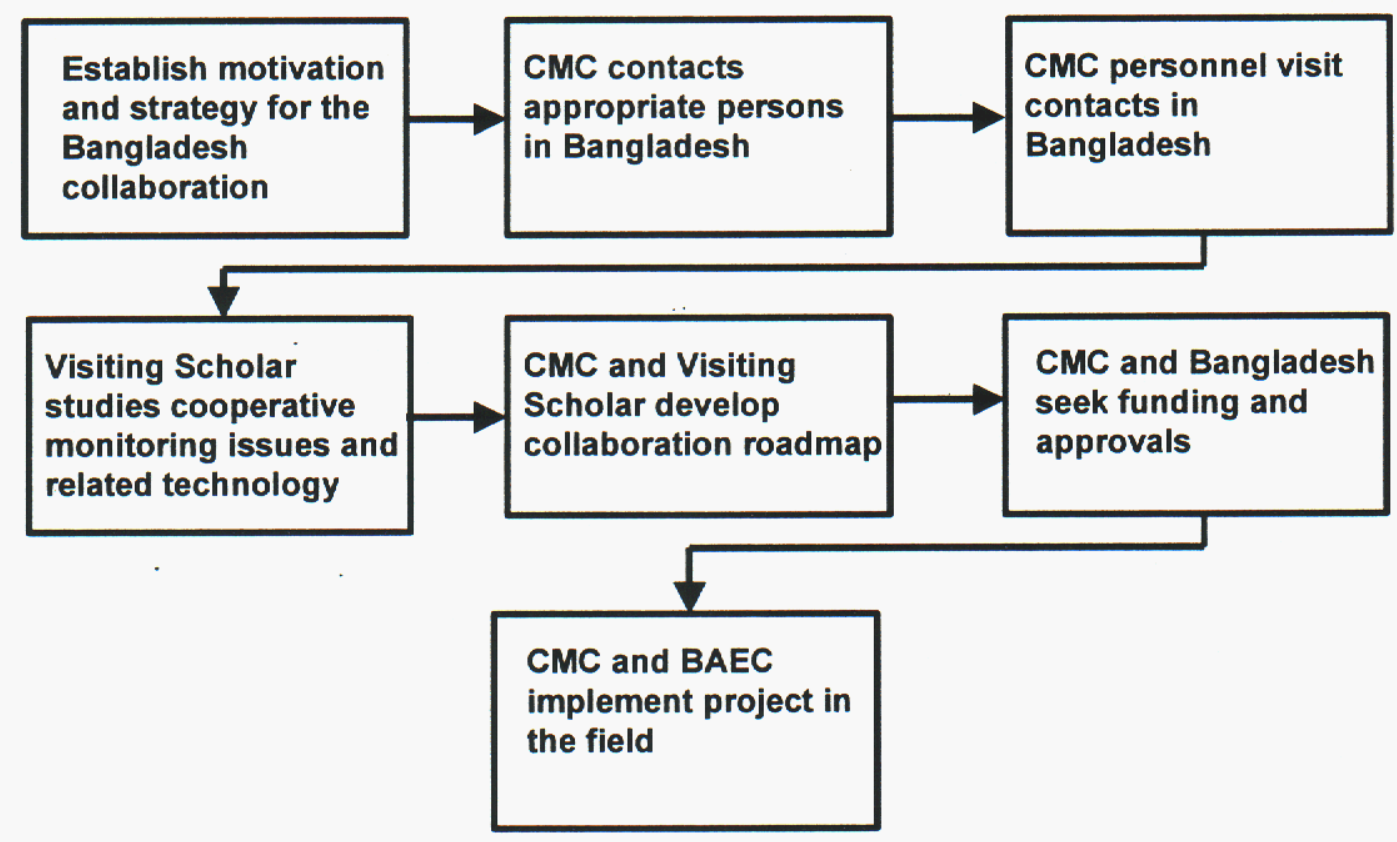

Figure 3: General Plan for CMC/Bangladesh Nuclear Cooperative Monitoring Implementation

A representative each from the CMC and the BAEC would take responsibility for implementing the project and perform point of contact duties.

An instrument such as a Memorandum of Understanding would be developed to delineate responsibilities. The work would proceed according to the work breakdown and milestones developed by the project team.

In general, the $\mathrm{CMC}$ would be responsible for all procurement outside Bangladesh. The BAEC would prepare technical specifications for required items. The BAEC would be responsible for all work at the site, including local transportation, construction, and availability of utility services at the designated sites. The CMC would support the BAEC on equipment commissioning and training. The CMC would help BAEC interface with the U.S. DOE's EML.

\subsection{Operation and Maintenance}

The BAEC would be responsible for operation and maintenance of the facilities provided under the present project and also for making information available for dissemination through the web site. The BAEC would designate a person to coordinate operation and maintenance activities under the project. 
The specific BAEC establishments that may be involved in the project are listed in the table below.

\begin{tabular}{|l|l|}
\hline \multicolumn{1}{|c|}{ Facility/lnstitute } & \multicolumn{1}{c|}{ Type of work } \\
\hline $\begin{array}{l}\text { Reactor Operation and Maintenance Unit, } \\
\text { Savar }\end{array}$ & $\begin{array}{l}\text { Non-proliferation } \\
\text { Fuel inventory and management } \\
\text { Reactor security and access control } \\
\text { Area monitoring }\end{array}$ \\
\hline Institute of Electronics, Savar & $\begin{array}{l}\text { Repair and maintenance } \\
\text { Design and fabrication of replacement items, etc. }\end{array}$ \\
\hline Electronics Division, AECD & Repair and maintenance \\
\hline Central Engineering Facility, Savar & Repair and maintenance (non-electronic) \\
\hline Engineering Division, BAEC Headquarters & Project implementation \\
\hline Nuclear Power and Energy Division, BAEC & Server \\
\hline $\begin{array}{l}\text { AECD (Chemistry and Accelerator } \\
\text { Divisions) }\end{array}$ & $\begin{array}{l}\text { Elemental analysis } \\
\text { Air quality monitoring (particulates) }\end{array}$ \\
\hline Health Physics Division, AECD & Analysis of environmental samples \\
\hline $\begin{array}{l}\text { Institute of Nuclear Science and } \\
\text { Technology, Savar }\end{array}$ & $\begin{array}{l}\text { Calibration } \\
\text { Environmental monitoring }\end{array}$ \\
\hline $\begin{array}{l}\text { Radiation Testing and Monitoring } \\
\text { Laboratory, Chittagong }\end{array}$ & Environmental monitoring \\
\hline Various BAEC Establishments & $\begin{array}{l}\text { Environmental sample collection } \\
\text { Maintenance of air sampling units }\end{array}$ \\
\hline
\end{tabular}

\subsection{Documentation and Reports}

Progress reports, procedures, manuals, final reports, and a variety of other reports are needed, as follows.

\section{Implementation phase:}

- Inception report

- Project implementation document

- Procedures and operating manuals

- Project completion report

\section{Operation and maintenance phase:}

- Interval reports on data and information

- Reports on performance and systems

The formats and contents of each category of the above reports would be decided through mutual consultation between the BAEC and the CMC. 


\subsection{Approval Process}

The approval process would proceed in parallel in the United States and Bangladesh. The approval process in the United States would involve CMC and the DOE. The CMC and the BAEC have briefed the DOE and the U.S. State Department on the need for the project and its impact on the South Asia region, overall objectives, rationale, project components, benefits, levels of effort, costs, and potential impact on non-proliferation.

In Bangladesh, the project would be initiated by the BAEC and sponsored by the Ministry of Science and Information \& Communication Technology (MOSICT). The reports would be formulated according to standard formats in two phases, namely

- Project Concept Paper, a distillation of the project plan, is intended to help assess the importance and priority of the project in the context of national plan, and

- Project Proforma, an extended version of the project, which provides the information for making the final decision on formal acceptance of the project. The decision is taken either at the level of the Planning Commission or the Executive Committee of the National Economic Council (ECNEC), depending on the size of the project in monetary terms.

Both documents would be reviewed by the responsible MOSICT group and then by a joint committee of the Planning Commission and relevant Ministries, before submission by the Planning Ministry or the ECNEC.

After approval, the project would be included in the Annual Development Program and financial inputs would be provided annually according to the investment profile specified in the approved project document. During this period, funding would be allocated from the Development Budget.

The Implementation Monitoring and Evaluation Division of the Planning Commission would examine the Project Completion Report. This exercise involves input-output balancing and assessment of performance in the implementation phase. This evaluation would form the basis for transfer of the completed project to the Revenue Head and annual recurring cost would be allocated annually according to the cost streams as given in the approved project document.

The CMC and the BAEC would collaborate by providing information that may be needed for preparing documents for obtaining all required approvals. 


\section{Conclusion}

The proposed project was developed with the ultimate goal of expanding the scope and extending the project to other countries in the region. The development of the project and its phasing recognizes possible limitations, such as availability of funds, local conditions, etc. One of the main considerations in developing the project was to ensure that in spite of such limitations, it could eventually grow into a nuclear transparency program involving other countries in the region. Ultimate success would be attained when all countries in the region feel motivated to join the project. Also, the project could become a model project that can be replicated in other regions such as North Africa, South Africa, etc.

The international community also has an interest in the success of regional and subregional activities. As the Bangladesh Nuclear Monitoring Project evolves and progresses, other agencies and related programs could become involved - for example, the IAEA through its Regional Cooperation Agreement among Asia-Pacific countries, and the CSCAP.

In conclusion:

- Taking a bilateral approach to begin the project could later be extended to the regional level to encourage nuclear transparency.

- In the future, the project could be considered as a model for implementation in other regions.

- Implementation of the project in phases and a wider participation by various countries would pave the way to the establishment of a regional cooperative monitoring center. 
Concepts for Cooperative Nuclear Monitoring in Bangladesh

This page intentionally left blank. 


\section{Appendix A. Evaluation Criteria}

This appendix contains example criteria that could be used for evaluation of projects and monitoring equipment proposed for the U.S.-Bangladesh Cooperative Monitoring Project. Each criterion will be ranked by priority and importance to the project.

\section{Evaluation of Project Components}

\section{Component 1}

\section{Required Criteria}

1. Conforms to existing obligations to non-proliferation and other agreements or protocols

2. Has capabilities and features that demonstrate and/or enhance regional nuclear transparency

3. Of interest to both $\mathrm{CMC} / \mathrm{DOE} / \mathrm{USG}$ and $\mathrm{BAEC} / \mathrm{MOSICT} / \mathrm{GOB}$

4. Acceptable to both $\mathrm{CMC} / \mathrm{DOE} / \mathrm{USG}$ and BAEC/MOSICT/GOB

5. Involves mutual commitment of $\mathrm{CMC}$ and $\mathrm{BAEC}$

6. Employs established technologies (proven, demonstrated in field, reliability, maintainability under local conditions, can be implemented and operated utilizing local infrastructure and capabilities, etc.)

7. Equipment is compatible with power and communications infrastructure, quality, and availability in Bangladesh

8. Requires readily available consumables, spare parts, etc. (preferably local, commercial)

9. Is compatible with environmental conditions in Bangladesh (e.g., temperature, humidity)

10. Can be implemented in a reasonably short time (e.g., months)

11. Collaborating institution in Bangladesh (with incremental addition of physical, financial and human resources) is capable of implementing, operating, and maintaining the equipment and systems

12. Facility is adequate for delivering data and information on a sustainable basis

13. Sustainable: has a long operating life with optimal operating cost

14. Has minimal potential negative consequences or effects, such as environmental hazards

\section{Desired Criteria}

1. Tangible spin-off benefits to the collaborating parties

2. Possibilities of involving other regional and international agencies in funding and/or management, including exchange of information and data on nuclear transparency

3. Capable of being extended in the future to a regional or international network

4. The system or equipment are cost-optimal

5. Expandable to additional nuclear monitoring applications

6. The system or equipment are a part of a comprehensive program on nuclear transparency and non-proliferation

7. Technologies can be transferred to and absorbed by the recipient country

8. Sandia National Laboratories can supply required equipment and systems 


\section{Component 2: Phase 1}

\section{Required Criteria}

1. Conforms to existing obligations to non-proliferation and other agreements or protocols

2. Has capabilities and features that demonstrate and/or enhance regional nuclear transparency

3. Of interest to both $\mathrm{CMC} / \mathrm{DOE} / \mathrm{USG}$ and BAEC/MOSICT/GOB

4. Acceptable to both $\mathrm{CMC} / \mathrm{DOE} / \mathrm{USG}$ and BAEC/MOSICT/GOB

5. Involves mutual commitment of $\mathrm{CMC}$ and $\mathrm{BAEC}$

6. Employs established technologies (proven, demonstrated in field, reliability, maintainability under local conditions, can be implemented and operated utilizing local infrastructure and capabilities, etc.)

7. Equipment is compatible with power and communications infrastructure, quality, and availability in Bangladesh

8. Requires readily available consumables, spare parts, etc. (preferably local, commercial)

9. Is compatible with environmental conditions in Bangladesh (e.g., temperature, humidity)

10. Can be implemented in a reasonably short time (e.g., months)

11. Collaborating institution in Bangladesh (with incremental addition of physical, financial and human resources) is capable of implementing, operating, and maintaining the equipment and systems

12. Facility is adequate for delivering data and information on a sustainable basis

13. Sustainable: has a long operating life with optimal operating cost

14. Has minimal potential negative consequences or effects, such as environmental hazards.

\section{Desired Criteria}

1. Tangible spin-off benefits to the collaborating parties

2. Possibilities of involving other regional and international agencies in funding and/or management, including exchange of information and data on nuclear transparency

3. Capable of being extended in the future to a regional or international network

4. The system or equipment are cost-optimal

5. Expandable to additional nuclear monitoring applications

6. The system or equipment are a part of a comprehensive program on nuclear transparency and non-proliferation

7. Technologies can be transferred to and absorbed by the recipient country

8. Sandia National Laboratories can supply required equipment and systems 


\section{Component 2: Phase 2}

\section{Required Criteria}

1. Conforms to existing obligations to non-proliferation and other agreements or protocols

2. Has capabilities and features that demonstrate and/or enhance regional nuclear transparency

3. Of interest to both $\mathrm{CMC} / \mathrm{DOE} / \mathrm{USG}$ and $\mathrm{BAEC} / \mathrm{MOSICT} / \mathrm{GOB}$

4. Acceptable to both $\mathrm{CMC} / \mathrm{DOE} / \mathrm{USG}$ and BAEC/MOSICT/GOB

5. Involves mutual commitment of $\mathrm{CMC}$ and $\mathrm{BAEC}$

6. Employs established technologies (proven, demonstrated in field, reliability, maintainability under local conditions, can be implemented and operated utilizing local infrastructure and capabilities, etc.)

7. Equipment is compatible with power and communications infrastructure, quality, and availability in Bangladesh

8. Requires readily available consumables, spare parts, etc. (preferably local, commercial)

9. Is compatible with environmental conditions in Bangladesh (e.g., temperature, humidity)

10. Can be implemented in a reasonably short time (e.g., months)

11. Collaborating institution in Bangladesh (with incremental addition of physical, financial and human resources) is capable of implementing, operating, and maintaining the equipment and systems

12. Facility is adequate for delivering data and information on a sustainable basis

13. Sustainable: has a long operating life with optimal operating cost

14. Has minimal potential negative consequences or effects, such as environmental hazards

\section{Desired Criteria}

1. Tangible spin-off benefits to the collaborating parties

2. Possibilities of involving other regional and international agencies in funding and/or management, including exchange of information and data on nuclear transparency

3. Capable of being extended in the future to a regional or international network

4. The system or equipment are cost-optimal

5. Expandable to additional nuclear monitoring applications

6. The system or equipment are a part of a comprehensive program on nuclear transparency and non-proliferation

7. Technologies can be transferred to and absorbed by the recipient country

8. Sandia National Laboratories can supply required equipment and systems 


\section{Component 3}

\section{Required Criteria}

1. Conforms to existing obligations to non-proliferation and other agreements or protocols

2. Has capabilities and features that demonstrate and/or enhance regional nuclear transparency

3. Of interest to both $\mathrm{CMC} / \mathrm{DOE} / \mathrm{USG}$ and BAEC/MOSICT/GOB

4. Acceptable to both $\mathrm{CMC} / \mathrm{DOE} / \mathrm{USG}$ and BAEC/MOSICT/GOB

5. Involves mutual commitment of $\mathrm{CMC}$ and $\mathrm{BAEC}$

6. Employs established technologies (proven, demonstrated in field, reliability, maintainability under local conditions, can be implemented and operated utilizing local infrastructure and capabilities, etc.)

7. Equipment is compatible with power and communications infrastructure, quality, and availability in Bangladesh

8. Requires readily available consumables, spare parts, etc. (preferably local, commercial)

9. Is compatible with environmental conditions in Bangladesh (e.g., temperature, humidity)

10. Can be implemented in a reasonably short time (e.g., months)

11. Collaborating institution in Bangladesh (with incremental addition of physical, financial and human resources) is capable of implementing, operating, and maintaining the equipment and systems

12. Facility is adequate for delivering data and information on a sustainable basis

13. Sustainable: has a long operating life with optimal operating cost

14. Has minimal potential negative consequences or effects, such as environmental hazards

\section{Required Criteria}

1. Tangible spin-off benefits to the collaborating parties

2. Possibilities of involving other regional and international agencies in funding and/or management, including exchange of information and data on nuclear transparency

3. Capable of being extended in the future to a regional or international network

4. The system or equipment are cost-optimal

5. Expandable to additional nuclear monitoring applications

6. The system or equipment are a part of a comprehensive program on nuclear transparency and non-proliferation

7. Technologies can be transferred to and absorbed by the recipient country

8. Sandia National Laboratories can supply required equipment and systems 


\section{Appendix B. Technical Specifications} the project.

This section contains technical specifications for the monitoring equipment desired for

\section{B-1. Equipment for Environmental Monitoring}

\section{HPGe Detector}

\section{- Crystal}

The detector will use a " $p$ " type coaxial intrinsic germanium crystal. The efficiency shall be at least $40 \%$ relative to a 3 "X3" NaI Crystal at $1.33 \mathrm{MeV}$ for a source-detector distance of 25 $\mathrm{cm}$. The energy resolution must be less than $2.0 \mathrm{KeV}$ at $1.33 \mathrm{MeV}$ and less than $1.4 \mathrm{KeV}$ at 122 $\mathrm{KeV}$. The ratio of the diameter of the crystal to its length shall be unity. This ratio shall not deviate from 1.0 by more than $20.0 \%$.

\section{- Preamplifier}

The preamplifier shall have diode FET protection. The detector shall use an in-line or slim-line preamplifier.

\section{- Cryostat}

The cryostat must have an integral, vertical dipstick. The end cap must have a length between 7.0 and 8.0 inches, and the diameter must be between 2.75 and 2.85 inches.

\section{Extra Low Background Requirements:}

- The cryostat shall have extra low background. It should be constructed from specially selected materials to minimize the presence of the naturally occurring radioisotopes.

- The end cap shall be made with magnesium.

- Detector cup, cooling rod, pedestal, and pedestal nut shall be made from OFHC copper.

- Cryostat flange shall be constructed using low cobalt, stainless steel.

- Molecular sieve shall be remote from the crystal.

- An aged lead shield of minimum thickness of 2 inches shall be placed between the detector and the preamp/HV filter.

\section{- Dewar} of its neck.

The dewar shall have a capacity of 30.0 liters. The dewar shall be $24 \pm 1$ inch at the top 


\section{- Liquid Nitrogen Monitor}

- The liquid nitrogen monitor must sense detector warm-up and provide a delayed shut down.

- The monitor must provide a signal for a visual and audible alarm.

\section{- Additional Requirements}

- The detector shall come with a minimum one-year warranty on all items. The supplier shall provide at least 5 years support for the detector with repair and spare parts availability.

\section{Liquid Scintillation Counters}

- Must be capable of separating alpha and beta events by the pulse shape discrimination method.

- Must provide dual MCAs with at least 4096 channels for separate display of alpha and beta spectra.

- Must be capable of automatic adjustment of the pulse shape discrimination setting to minimize the alpha to beta and beta to alpha spillovers. The alpha/beta spillover shall be less than $1.0 \%$.

- Must provide and save spillover curves as well as displaying them on screen.

- Must have an external low energy gamma source to monitor sample quenching by analyzing the Compton spectrum in the sample.

- Must have an automatic sample changer with a capacity of at least 200 samples. The sample changer shall be capable of handling 20 and $7 \mathrm{ml}$ vials.

- Must have a monitor to check for sample heterogeneity. It shall flag all nonhomogeneous samples.

- Must be operated by an IBM PS/2 computer with at least $2 \mathrm{MB}$ of RAM, $20 \mathrm{MB}$ of hard disk, one 3.5" high density (1.44 MB) floppy, color high-resolution monitor, and DOS operating system.

- The interface program must be capable of writing the spectral data, region of interest(s), quench information, protocol and other relevant data to a custom formatted ASCII file.

- The interface program shall be capable of executing a user-defined routine, such as a batch file, at the end of each counting batch.

- Must have a live display of spectral data with regions of interest identified. The display shall also show the live cpm values for each region of interest.

- Must be capable of optimizing the region of interest for a given isotope as to maximize the figure of merit $(\mathrm{E} 2 / \mathrm{B})$.

- Must have an automatic quality control program to check the detector performance. The performance checks shall follow the efficiencies and backgrounds of $\mathrm{H}-3$ and $\mathrm{C}-14$. There must be an algorithm to perform a statistical check and issue a warning if a parameter is out of bound.

- The system must be able to automatically monitor and adjust the Photo multiplier gains. 
- The counting system shall be composed of two photo multipliers operating in coincidence or singly as selected by the user.

- Must have an extra low background rate. It shall use a time resolved spectroscopy method to filter out the background events. The optimized region for $\mathrm{H}-3$. shall have a background rate of less than $4.0 \mathrm{cpm}$ with a minimum of $20.0 \%$ counting efficiency.

\section{Alpha Spectroscopy}

\section{- Hardware}

- The Alpha Spectroscopy system shall consist of one unit with twenty-four (24) independent vacuum chambers.

- The plumbing for the vacuum system shall be built in to the unit. A vacuum pump shall be included with the system.

- Each vacuum chamber shall have its dedicated built-in HV supply, preamplifier, amplifier, and a $4 \mathrm{~K}$ ADC.

- Each vacuum chamber shall be equipped with a door status sensor to determine whether the chamber door is open or close. There would be an option to turn the HV off when a door in opened.

- Each chamber shall have a rugged ion-implanted silicon detector with a surface area of $500 \mathrm{~mm}^{2}$ and an alpha resolution of $20 \mathrm{KeV}$. The detectors shall have rear microdot connectors.

- The unit must be equipped with an electronic Cartesian diver system to hold the vacuum at a set point. A back bias shall be provided for each of the eight sample holders.

- The system shall come with all the necessary components (e.g., interface card and cables) to interface the unit with an IBM AT-compatible computer.

\section{- Software}

- The vendor shall provide integrated software for automated hardware control, data acquisition, data analysis, and quality control checks.

- The software must run on Windows operating system.

- The software must be capable of controlling the vacuum level, detector bias, preamplifier and amplifier settings, ADC, and MCA functions.

- The software shall include a quality control package to track background, peak centroid and count rate and FWHM for a designated source, amplifier gain, and detector bias.

- The data acquisition part of the software would allow simultaneous or individual operation of the eight channels.

- The analysis program shall be an ROI-based routine capable of tracer and tracerless analyses.

- The output report format must be modifiable by the user.

- The output report shall have the option of reporting the hardware settings, ROI information, isotope activities, 2-sigma errors, MDA values, critical level values, spectrum graphs, and a summary of the QC data. 


\section{- Additional Requirements}

- The system shall come with a minimum one-year warranty on all items.

- The supplier shall provide at least 5 years support for the system with repair and spare parts availability, and software.

- The supplier shall modify the software, if necessary, to help customize the package.

\section{Air Monitoring}

\section{- PM10 MASS Flow Controller}

In 1971, The Environmental Protection Agency (EPA) promulgated primary and secondary national ambient air quality standards for particulate matter measured as "total suspended particulate" or "TSP". In accordance with the Clean Air Act, the EPA reviewed and revised the health and welfare criteria upon which these primary and secondary particulate matter standards were based.

On March 20, 1984, the EPA proposed changes in the standards based on its review and revision of the criteria. On July 1, 1987, the EPA promulgated its final decisions regarding these changes. The final decisions include:

1. Replacing TSP as the indicator for particulate matter for the ambient standards with a new indicator that includes only those particles with an aerodynamic diameter less than or equal to nominally ten (10) microns (PM10).

2. A new Federal Reference method for measurement of PM10 in ambient air. The Reference method for the measurement of atmospheric particulate matter is known as PM10 and is based on selection of PM10 particles by internal separation, followed by filtration and gravimetric determination of PM10 mass on the filter substrate.

\section{- PM10 Volumetric Flow Controller}

The Volumetric Flow Controller (VFC) is a dimensional venturi device used to control gas flow. When applied to a high volume air sampler, this flow control principal incorporates a smooth-wall venturi orifice that gradually opens to a recovery section. Vacuum is provided by a motor downstream of the venturi. Over $95 \%$ of the energy lost in differential pressures across the restricting orifice is recovered in this design.

Flow control is accomplished by occluding or restricting and thus accelerating the airflow through the venturi at some point in the flow stream, the air velocity would equal the acoustic velocity or speed of sound, and critical flow would be achieved. As long as downstream changes are small, all conditions at the venturi (including the flow rate) are determined by upstream conditions. This condition is referred to as "chocking" and is a distinctive characteristic of all VFCs. The GMW PM10/VFC utilizes this principle of choked or critical flow to maintain a 
constant actual flow rate of 40 ACFM (1.3ACMM) over the sample period. Since critical flow through the venturi is not greatly affected by changes in the filter loading, ambient temperature or barometric pressure, a stable volumetric flow rate is maintained as long as power is provided to the sampler blower motor.

The orifice used in this system can also be described as a well-documented Critical Venturi Meter (CVM). Basically, a CVM is a specially machined nozzle or restriction device designed to react to a specific pressure ratio expressed in absolute terms. When air reaches the speed of sound in the throat (smallest diameter) of the CVM, a sound pressure barrier is set up that would not allow more air through under the existing temperature and pressure conditions. This is the "critical flow" point of the meter, thus the name Critical Venturi Meter. Simply put, the Volumetric Flow controller regulates flow at a constant but non-adjustable rate without any moving parts or electronic components. GMW PM10 Samplers bear an identification label with the FRM designation number RFPS-1287-063.

\section{- Datalogger and Transceiver for Airborne Environmental Measurements}

A variety of meteorological instruments can be combined with background radiation monitoring for making airborne environmental measurements. The integrating component is a "datalogger," such as the Model CR23X by Campbell Scientific. The datalogger can collect information from a barometric sensor, rain gage, wind speed and direction indicators, and temperature and humidity probes. A radiation sensor, such as a compensated Geiger-Mueller detector or $\mathrm{NaI}$ scintillator with associated electronics, would provide the ambient radiation measurement. A satellite transceiver with integrated Global Positioning System (GPS) sensing, connected to the datalogger, would provide the remote communications link from wherever the airborne monitoring station is deployed.

\section{B-2. Facility and Material Monitoring}

\section{Baseline Components}

JContainment and Surveillance (C\&S) technologies are used for monitoring system integrity and item counting. Sensors may include loop seals, identification tags, contact closure detectors, glass break sensors, passive infrared intrusion detectors, and object motion sensors.

The loop seals and motion sensors provide access and containment monitoring. Identification tags provide continuous in-place monitoring of items. An independent camera system can capture images and views when triggered by a sensor or using interval recording. Sensors should have built-in features such as a sophisticated state-of-health message protocol and tamper indication.

\section{Special Nuclear Material (SNM) Attribute Measurement}

Four types of radiation sensors for measuring the U235 enrichment are available:

\section{- Rad-Couple Gamma Radiation Sensor (RF)}


Rad-Couple sensor is a very low-power consumption device that produces an electrical current proportional to the amount of gross gamma irradiation present. The sensor operates in a wireless (battery operated) configuration. Rad-Couple provides a gross gamma measurement when placed near radioactive material. Rad-Couple sensors may be configured into the AIMS $\mathrm{RF}$ network and installed to monitor each item. The sensors report gross gamma measurement (implying continuous presence and location of a material), nod tamper, and $\mathrm{SOH}$ via $\mathrm{RF}$ to the data Acquisition System.

\section{- Rad-Couple Gamma Radiation Sensor (Hardwired)}

Rad-Couple sensor with a hardwired configuration can monitor each item and report gross gamma measurement (implying continuous presence of a material), node tamper, and $\mathrm{SOH}$. These may be interfaced with an Echelon LonWorks (Local Operating Networks) communication interface incorporated into its design.

\section{- Rad-SIP Gamma Radiation Sensor}

Rad-SIP is a gamma irradiation sensor that uses a silicon PIN diode as a sensing unit. It can determine the relative U235 enrichment from uranium materials. Signal levels from this sensor are selected by a pulse height discriminator for precise gamma energy monitoring of U235. This sensor can monitor each item and can be interfaced as in the earlier case. The sensor reports gross enrichment (implying continuous presence of a container of material), node temper, and $\mathrm{SOH}$.

\section{- Fast Gamma Radiation Sensor}

The Fiber-Optic Attribute Sensor Technology (FAST)/ Gamma Radiation Sensor is a plastic scintillator coupled via a fiber optic link to a two-dimensional photo-multiplier tube, which can simultaneously monitor hundreds of sensors. The plastic scintillator is a sensitive, self-powered gamma ray detector that requires no electrical excitation for the continuous monitoring of SNM in storage. The sensor provides a gross gamma measurement when placed near radioactive material.

For the Fast system, only the passive components may be placed in the radiation field; all electronic components may be removed from the potentially hazardous environment and placed where they could be readily accessed (e.g. outside the field in the secure cabinet with the on-site data acquisition system). Its dedicated computer, interfaced with the on-site Data Acquisition System, may be used for monitoring.

\section{On-Site Sensor Network}

The alternative technologies for local network is as follows:

- Server Digital Image Surveillance System (SDIS)

The Server Digital Image Surveillance System (SDIS) is a system for collecting data from attached sensors, storing the data, and then sending the data to authorized recipients. The 
SDIS communications server, which the IAEA often uses in remote monitoring for international Safeguards, is a commercial product from Canberra Aquila, Inc. The system can collect a variety of data, including instrumentation signals, video from attached cameras, intrusion detection and motion sensors, material seals, door switches, and many others. The computercontrolled acquisition system stores data locally on dual hard disk drives. Software uses dual telephone modems to automate copying the data to desired remote locations as required. Mains power is supported by an uninterruptible power supply. The unit is modular, fully enclosed and sealed for tamper indication.

\section{- Echelon LonWorks}

Echelon LonWorks is a Local Operating Network developed for the distributed control systems. It is a complete platform for implementing control network systems. These networks consist of intelligent devices or nodes that interact with their environment, and communicate with another over a variety of communications media using a common, message-based control Protocol (LonTalk protocol). The communications media support includes twisted pair, AC power line, and link power, etc. Free topography network configuration may be used, which allows for the connection of sensors in a bus, star, ring, daisy chain, or mixed architecture. LonWorks has a built-in communication protocol that allows for peer-to-peer communications with or without authentication of messages. For instance, the peer-to-peer link may be used for the sensor trigger to the ICAM. The LonTalk protocol supports interoperability at both node and product levels. LonWork provides reliable, error-free communications within the sensor network of Remote Monitoring System (RMS).

\section{Data Acquisition System (DAS)}

The DAS provides local storage and backup of the data and is the interface between the sensor network and remote data review sites. Its main function is collecting and archiving data. It can provide the following functions:

- Interface to the sensor network

- Time synchronization of ICAM, Master Data Node (MDN) and DAS computer

- Database logging of sensor events and auxiliary data

- SOH interval monitoring and logging

- Local storage and backup of data

- Limited real time display of data

- Off-site communications interface

The interface to the sensor network may be via the MDN. The MDN provides a simple serial communications protocol for the transfer of sensor messages and image files from the MDN to DAS. Each day (say at midnight), the DAS can archive the preceding day's data and authenticate the data. After archiving the preceding day's data, the DAS can synchronize the time both on MDN and the ICAM to the DAS time. The DAS should maintain a history of each sensor and the last time data was received. If a programmable period of time passes without a message from the sensor, the DAS logs missing $\mathrm{SOH}$ information for that sensor. Messages 
about start, stop, restart, and continue modes of acquisition of the DAS application can also be logged.

The MDN is the point of interface between the sensor network and the DAS computer. Primary functions of the MDN may include sensor message authentication, date- and timestamping of events, temporary storage of data, and transfer of sensor data to the DAS computer for local storage and archiving. The MDN is a commercial product (Coactive Aesthetics Inc. of Sausalito, California.

\section{Data Transmission}

The data transmission from DAS to the DIRS may be accomplished in two methods:

- A public access telephone link, and

- A dedicated satellite link.

\section{Telephone line}

A telephone line may be used for transfer of data from DAS to the DIRS at the remote facilities. The DAS can have a Hayes Accura $28.8 \mathrm{~kb} / \mathrm{s}$ external modem configured for automatic transfer rate selection dependent upon the landlines status. (Each facility has to have its own modem at each DIRS).

\section{Data and Image Review System (DIRS)}

The DIRS application provides the following basic functions:

- Communications with the DAS

- Data transfer

- Storage and archive of the database

- Graphical User Interface

- Review of sensor and image data

- Event and image printouts

Data may be further reduced using filters based upon either sensor type or message level. 
Message levels are:

- Material alarm: Alarms that indicate a potential access to the material

- Material Message: Status-type message of the material, e.g., container position or NDA sample reading

- System Alarm: Alarms indicating abnormal operation, e.g., sensor on- or off-line

- System Message: Maintenance-type messages, e.g., begin/continue acquisition, transparent maintenance

\section{Performance criteria:}

Criteria for equipment in a $\mathrm{C} \& \mathrm{~S}$ monitoring system are as follows:

\section{$\underline{\text { Sensors }}$}

- Detect all seal openings and closings

- Detect all tamper events

- Do not generate false detections

- Generate an image trigger for all seal alarms

- Explain all messages

- Receive all state of health messages

\section{Motion sensors}

- Detect all motion started and motion ended events

- Detect all tamper events

- Do not generate false detections

- Generate a video trigger for all motion-started alarm

- Explain all messages

- Receive all SOH messages

\section{$\underline{\text { Video }}$}

- Display adequate image quality

- Associate each image with a sensor trigger

- Generate clear images

- Authenticate all image files

- Explain all image files

\section{$\underline{\text { RPU/LON }}$}

- Transmit all sensor messages correctly

- Detect all missing SOH messages

- Authenticate all RF and other network messages

- Explain all network messages

- Transmit all video files correctly

- Transmit correctly all network management messages 


\section{Data Acquisition System (DAS)}

- Time-tag all network messages correctly

- Indicate tampers to the DAS-Master Data Node

- Authenticate all messages correctly

- Store all network messages correctly

- Display all network messages correctly

- Transmit all configuration commands correctly

- All network messages delayed one hour by the data logger

- Key management worked correctly

$\underline{\text { Data Communication }}$

- Provide telephone Communication

- Provide correct operation

- Provide correct modem remote communication software

- Correctly transmit all files

- Provide daily telephone access

Data and Image Review Station (DIRS)

- Store all files correctly

- Display all data and images correctly

- Print all data and images correctly

- Archive all data and images correctly

- Filter data correctly

- Provide effective and efficient data review

- Provide adequate user documentation 


\section{About the Author}

C. S. Karim joined the Bangladesh Atomic Energy Commission as a Senior Scientific Officer in 1977. In 2000, he was appointed as a Member of Physical Science of BAEC. He is also responsible for the Nuclear Power Program of Bangladesh and is the Technical Focal Point for the Comprehensive Test Ban Treaty Organization. His primary field of interest is nuclear power, energy economics, and energy planning. As a member of the Physical Science of BAEC, he is responsible for top-level supervision of the R\&D activities in the disciplines of physical science. He also participated in the formulation of National Energy Policy of Bangladesh. He was a member of the Bangladesh Delegations to the Annual General Conference of the International Atomic Energy Agency (1979 through 2001). He obtained his B.Sc. (Honors) in Physics in 1969, his Masters of Science in Physics in 1972, and received his Ph.D. in Nuclear Engineering in 1976. 
Concepts for Cooperative Nuclear Monitoring in Bangladesh

\section{Distribution}

350 MS 1373 CMC Library, 5341

1 MS 9018 Central Tech Files, 8945-1

2 MS 0899 Technical Library, 9616

1 MS 0619 Review \& Approval Desk, 9612 